\title{
Magnetocaloric materials: From micro- to nanoscale
}

\author{
João H. Belo ${ }^{\text {b) }}$ \\ IFIMUP and IN - Institute of Nanoscience and Nanotechnology, Departamento de Física e Astronomia da \\ Faculdade de Ciências da Universidade do Porto, Porto 4169-007, Portugal; CICECO - Aveiro Institute of \\ Materials and Department of Physics, University of Aveiro, Aveiro 3810-193, Portugal \\ Ana L. Pires, ${ }^{\text {b) }}$ João P. Araújo, and André M. Pereira ${ }^{\text {a) }}$ \\ IFIMUP and IN - Institute of Nanoscience and Nanotechnology, Departamento de Física e Astronomia da \\ Faculdade de Ciências da Universidade do Porto, Porto 4169-007, Portugal
}

(Received 16 June 2018; accepted 24 August 2018)

Twenty one years ago, the discovery of the giant magnetocaloric effect (GMCE) at room temperature completely revolutionized the magnetocaloric materials field demonstrating the potential of magnetic refrigeration at room temperature and setting the beginning of a race for the best magnetocaloric material. Since then, hundreds of different bulk magnetic materials were studied in detail; however, only a small set of these exhibit GMCE. In the last ten years, the broad interest on these materials leads to the extension of their study to the micro- and nanoscale. In this review, we highlight the main motivations for exploring the size-reduction both from the technological and the purely scientific point of view and stress the general consequences on the magnetic and magnetocaloric properties. The emergence of different underlying mechanisms driving these effects will be identified with particular emphasis for the set of materials presenting GMCE.

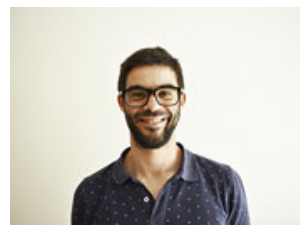

João H. Belo

João Horta Belo is a Post-doc researcher at CICECO - Universidade de Aveiro, Portugal. He finished his Ph.D. in July 2017 at IFIMUP-IN, Universidade do Porto. His research covered both magnetocaloric theoretical modeling and the study of the size reduction effects on the $\mathrm{R}_{5}(\mathrm{Si}, \mathrm{Ge})_{4}$ multifunctional materials. He has collaborated with Imperial College London, Ames Lab and Instituto de Nanociencia de Aragon and he is an active user of large facilities as ESRF, Max Lab and HFML. In 2014, he was awarded the ICTF16 IUVSTA-Elsevier Student Award. He is actively involved in several scientific dissemination activities (IOP Nanotechweb.org). In resume, Belo is fascinated by the hidden potential of micro/nanostructuring magnetovolume-coupled materials.

\section{INTRODUCTION}

Magnetic cooling systems at room temperature have emerged as an alternative to the conventional refrigeration technology due to two breakthrough discoveries at the end of the 1990s: the magnetic refrigerator prototype developed by Zimm et al. ${ }^{1}$ in 1998 and the giant magnetocaloric effect (GMCE) discovery by Pecharsky and Gschneider reported in $1997 .^{2}$ These milestones led to a remarkable increase in the number of room temperature magnetic refrigerator prototypes developed per year and exponential interest in the search for optimized magnetocaloric materials because it offered the possibility of using green energy, compared to today's conventional cooling systems such as air conditioners and freezers. ${ }^{3,4}$ One of the key points of this technology is the magnetic material since the GMCE is associated with

\footnotetext{
a) Address all correspondence to this author.

e-mail: ampereira@fc.up.pt

b) These authors contributed equally to this work.

DOI: $10.1557 /$ jmr.2018.352
}

a first-order transition, arising from the strong spin-lattice interplay thus leading to a strong magnetovolume exhibited by these materials. ${ }^{5-7}$ Till now, several families of compounds exhibiting the magnetocaloric effect (MCE) near room temperature were discovered, such as $\mathrm{Fe}-\mathrm{Rh},{ }^{8}$ Gd-Si-Ge, ${ }^{2} \mathrm{La}-\mathrm{Fe}-\mathrm{Si}^{9}$; Ni-Mn-Ga, ${ }^{10} \mathrm{Mn}-$ $\mathrm{As},{ }^{11}$ and manganites systems. ${ }^{12}$ Recently, another material was found, Eu2In, exhibiting remarkable magnetocaloric properties, despite its still low operational temperature. ${ }^{13}$ Since their discovery, there has been a constant effort to understand how to improve their magnetocaloric properties. ${ }^{14-18}$ As the prototype development and optimization continue, other challenges have appeared such as (i) the reduction of the heat exchange time between the material and the heat exchanger ${ }^{3}$; (ii) smart thermal control via integrated thermal switches ${ }^{19,20}$; (iii) diminish the undesirable magnetic hysteresis loss ${ }^{15,21}$; (iv) increase the mechanical stability of the magnetic materials in order for them to be able to undergo hundreds of thousands of cycles and ${ }^{22-24}$; (v) increase the materials MCE for low-applied field
(C) Materials Research Society 2018. This is an Open Access article, distributed under the terms of the Creative Commons Attribution licence (http://creativecommons.org/licenses/by/4.0/), which permits unrestricted re-use, distribution, and reproduction in any medium, provided the original work is properly cited. 
values $^{25}$; (vi) expanding operational temperature range by exploring further the study of cascade systems composed of several magnetocaloric materials. On materials development, the main strategies that have been adopted to cope with these challenges are chemical stoichiometry tuning, multistimulus (pressure, magnetic field, and temperature) and the size-reduction. The latter is the most unexplored strategy in this field. It is important to remark that the micro- and nanostructuring advantageous features could be brought to the macroscale by following smart bottom-up assembling strategies. In this review, we will focus on the latter by highlighting the most relevant breakthroughs on the field. The remarkable effort, intensified in the last years, to study magnetocaloric materials, or more generally materials with strong magnetovolume coupling, at the micro- and nanoscale is motivated not only by the need to solve the above-mentioned challenges in macroscopic magnetic refrigeration applications but also for opening new avenues on exploring these effects on innovative micro- and nanodevices. From the purely scientific interest, there are several fundamental questions deserving highlight: (i) the existence/ absence of a critical size below which the magnetovolume coupling vanishes; (ii) impact of the symmetry (magnetic, electrical, etc.) break at the nanostructure surface; (iii) the materials atomic structure stabilization; (iv) interface film-substrate and core-shell effects; (v) the impact of intrinsic surface stress in nanoparticles; (vi) the effects of introducing micron-sized pores; (vii) the consequences of changing the grain size; (viii) the repercussion in the transition kinetics, among others.

Concerning the technological applications, the role played by the size-reduction on the engineering of innovative devices at the micro- and nanoscale is still in its early age. As fundamental research continues to further develop, novel devices/ideas are expected to arise rapidly, including microrefrigerators, ${ }^{26-28}$ thermal switches, ${ }^{19,20}$ microfluidic pumps, ${ }^{29-31}$ energy harvesting devices, ${ }^{32}$ or even at the biomedical applications such as hyperthermia or drug delivery. ${ }^{33}$

In the past few years, the size-reduction efforts were reviewed by different authors. Each author has focused on one particular micro or nanostructure, as the case of the thorough review dedicated to the development of magnetocaloric thin films by Miller and coworkers, were they stress the necessity of smart engineering to take full advantage of the miniaturization novel opportunities. ${ }^{34}$ Franco and coauthors have dedicated a section to the nanostructured materials on their general materials review, where the importance of correct assessment of the materials properties at the microand nanoscale is addressed. ${ }^{35}$ More recently, Lyubina illustrated the importance of the magnetocaloric thin film development, in particular, in their potential to change the way the magnetocaloric materials are applied in a system. ${ }^{36}$ Alternatively, magnetocaloric nanoparticles have been attracting considerable attention due to their various application prospects as identified by Tishin and coworkers in their review report. $^{33}$

In this review, the goal is to give the reader a general overview of the miniaturization (from micro- to nanoscale) of different structures (microparticles, nanoparticles, nanowires, nanorods, and thin films) of the most important magnetocaloric materials families. As going along the groundbreaking results published so far, the major challenges that have been overcome and those that still need to be tackled will be identified. We have decided to highlight innovative results considering different points of view: unique production methods, microand nanoscale characterization techniques, and the discovery of unexpected results obtained in simple and complex systems. All the mentioned results will be compared with their respective bulk counterparts.

This review is divided by sections dedicated to different material families and where at least two different micro/nanostructures are covered.

\section{MAGNETOCALORIC EFFECT}

Before deepening onto the different materials microand nanostructure, a brief description of the MCE should be given. The MCE has a 2-fold definition: (i) it is a temperature change undergone by a magnetic material when a field is applied in an adiabatic process $\left(\Delta T_{\mathrm{ad}}\right)$ and (ii) it is an entropy change of a magnetic material when a magnetic field is applied in an isothermal process $\left(\Delta S_{\text {iso }}\right)$. According to thermodynamics, magnetic materials can be thought of as systems comprising three main energy reservoirs: the phonons, as the vibrational excitations of the lattice, the magnetic states of the magnetic sublattice, and the electronic contribution associated with the electron bands of the system. In the thermodynamics formalism, the total entropy of a magnetic system at constant pressure can be written as $S_{\text {tot }}(T, H)=$ $S_{\mathrm{L}}(T, H)+S_{\mathrm{M}}(T, H)+S_{\mathrm{E}}(T, H)$, where $S_{\mathrm{L}}(T, H)$, $S_{\mathrm{M}}(T, H)$, and $S_{\mathrm{E}}(T, H)$ are the lattice, magnetic, and electronic contributions to the total entropy $S(T, H)$, for a specific temperature and magnetic field. Hence, the MCE is intimately related to the interplay and energy flow between these three kinds of excitations. To minimize the total energy, the material magnetic moments tend to re-orientate themselves along the direction of the applied magnetic field, thus decreasing the entropy associated with the magnetic sublattice (from a disordered to an ordered state). If this process occurs under adiabatic and reversible conditions, the system must compensate the decrease of magnetic 
entropy $\left(S_{\mathrm{M}}\right)$ by, typically, increasing the lattice entropy $\left(S_{\mathrm{L}}\right)$ to maintain the total entropy constant. Thus, an entropy (energy) transfer occurs from the magnetic to the lattice reservoir, i.e., an increase in the material temperature occurs. This temperature change is experimentally mensurable and is identified as $\Delta T_{\mathrm{ad}}$. On the other hand, if the magnetic field is applied in an isothermal process, where there are heat transfers to the surroundings and hence there are no constraints to the total entropy, the change in the magnetic entropy will, typically, lead to a change of the system entropydefined as $\Delta S_{\text {iso. }}$. Hence, MCE is characterized both by $\Delta T_{\text {ad }}$ and $\Delta S_{\text {iso }}$, which can be expressed via Maxwell relations, as

$$
\begin{gathered}
\Delta S_{\text {iso }}(T, H)=\int_{H_{\mathrm{i}}}^{H_{\mathrm{f}}}\left(\frac{\partial M(T, H)}{\partial T_{H}}\right)_{H} \mathrm{~d} H, \\
\Delta T_{\mathrm{ad}}(T, H)=-\int_{H_{\mathrm{i}}}^{H_{\mathrm{f}}} \frac{T}{C_{\mathrm{p}}}\left(\frac{\partial M(T, H)}{\partial T_{H}}\right)_{H} \mathrm{~d} H,
\end{gathered}
$$

where $M$ represents the magnetization and $C_{\mathrm{p}}$ represents the specific heat. Typically, most reports publish $\Delta S_{\text {iso }}$ data instead of $\Delta T_{\mathrm{ad}}$, as the former is relatively easier to measure with standard magnetometers (although a detailed protocol should be followed ${ }^{37,38}$ ). $\Delta T_{\text {ad }}$ measurements are even more challenging at the micro- and nanoscale due to the technical difficulties to guarantee an adiabatic procedure and to the rapid thermal diffusion from the studied micro/nanostructure toward a heat/cold sink (such as the substrate in a thin film-substrate system). However, as can be noticed, both entities are strongly dependent on the materials magnetization temperature dependence curve, in particular, in its derivative. As will be seen, this fact has a major influence on the $\mathrm{MCE}$ at micro/nanostructures.

Furthermore, another parameter was defined by Pecharsky and Gschneidner ${ }^{39}$ to help estimate materials performance - the refrigerant capacity $(q)$, which is a measure of how much heat can be exchanged between the cold and the hot sink of an ideal refrigerator:

$$
q=\int_{T_{\mathrm{c}}}^{T_{\mathrm{h}}} \Delta S(T, P, \Delta H) \mathrm{d} T
$$

which can be approximated by a simple product:

$$
\mathrm{RCP}=\Delta S^{\max } \cdot \text { FWHM },
$$

of the entropy change peak $\left(\Delta S^{\max }\right)$ and the full width at half maximum (FWHM) of the $\Delta S(T)$ curve giving the relative cooling power (RCP).

\section{MAGNETOCALORIC SYSTEMS}

\section{A. Single elements: Rare-earths and others}

Gadolinium $(\mathrm{Gd})$ presents a strong magnetovolume coupling, high magnetic moment at room temperature and is considered the benchmark material for room temperature magnetic refrigeration. For these facts, $\mathrm{Gd}$ micro- and nanostructures are thought to pave the way for scale-reduction magnetocaloric research. Miller's group has been performing a thorough study on the influence of different thin film growth and preparation techniques on the structural, magnetic, and magnetocaloric properties of Gd films. ${ }^{34,40}$ Generally, they have found major differences on the film behavior when comparing to bulk: a significant reduction of the MCE (in particular the maximum magnetic entropy change, $\Delta S_{\mathrm{m}}{ }^{\max }$ ), a broadening of the $\Delta S_{\mathrm{m}}(T)$ curve and a reduction of the temperature at which the $\Delta S_{\mathrm{m}}$ reaches its peak. For $30 \mathrm{~nm} \mathrm{Gd}$ thin films grown by RF-sputtering onto $450{ }^{\circ} \mathrm{C}$ preheated $\mathrm{Si}$ oxidized substrates, Miller and coworkers have found that pregettering the sputtering chamber constitutes an important step toward the improvement of the magnetocaloric properties, namely, the maximum magnetic entropy, $\Delta S_{\mathrm{m}}{ }^{\max }$, which is increased by $\sim 50 \%$, the RCP or refrigerant capacity (RCP) by $\sim 30 \%$ (as can be seen in Fig. 1) and the overall saturation magnetization value by $\sim 35 \%$. Such improvements are mostly attributed to the lower $\mathrm{Gd}$ oxide content and to an improved film roughness and morphology.

Other interesting studies on Gd thin films are devoted to the dependence of their Curie temperatures $\left(T_{\mathrm{C}}\right)$ on the thin film growth details. In the literature, a wide range of

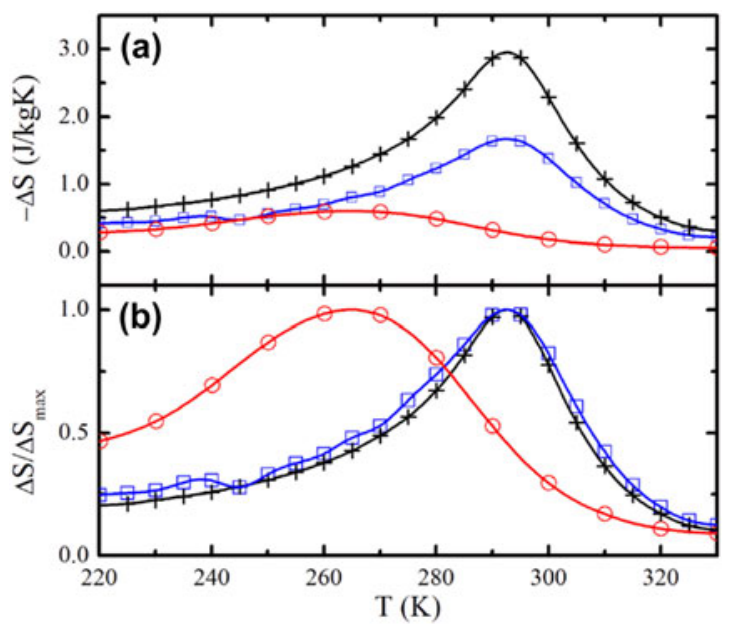

FIG. 1. (a) $\Delta S_{\mathrm{m}}(\Delta H, T)$ for a $30 \mathrm{~nm}$ thick Gd film grown at room temperature (red circles), at $450{ }^{\circ} \mathrm{C}$ after the gettering process (blue squares) and bulk Gd (black crosses) (b) Same data as in (a) but normalized to $\Delta S_{\mathrm{m}}{ }^{\max }$. Reprinted with permission from Ref. 34. Copyright 2014 American Vacuum Society. 
$T_{\mathrm{C}}$ values can be found, ranging from 260 to $295 \mathrm{~K}$ (which is approximately bulk Gd $T_{\mathrm{C}}$ ). ${ }^{41-44}$ For polycrystalline thin films, such $T_{\mathrm{C}}$ distribution can be directly associated with the crystallite size distribution present in the film. However, there are other factors playing an important role, such as the effect of $\mathrm{H}_{2}$ content and substrate temperature $\left(T_{\mathrm{S}}\right)$ on the properties of thin (50 nm thick) Gd films grown by DC sputtering. ${ }^{44}$ In contrast to what happens when $\mathrm{N}$ atoms penetrate interstitially the Gd lattice, compressing it and leading to a decrease of $T_{\mathrm{C}}$, it was observed that in fact the $T_{\mathrm{C}}$ increases with raising $T_{\mathrm{S}}$ (higher $T_{\mathrm{S}}$ promotes higher $\mathrm{H}$ absorption) up to a limit, after which it did not increase further. Especially, the film prepared at $T_{\mathrm{S}}=600{ }^{\circ} \mathrm{C}$ was found to have a $T_{\mathrm{C}}$ very close to the bulk value $(292.5 \mathrm{~K})$, where the good crystallinity and the $c$-axis preferential orientation were pointed as causes for this behavior. Moreover, Scheunert and coworkers ${ }^{41}$ have grown Gd thin films $(50 \mathrm{~nm})$, sandwiched into two Ta $(5 \mathrm{~nm})$ capping and seeding layers, by DC sputtering under ultrahigh vacuum conditions (base pressure $<10^{-9}$ mbar) and have studied the influence of the growth rate and also $T_{\mathrm{S}}$ on the magnetic properties of these films. They have shown that a $1.23 \AA / \mathrm{s}$ growth rate toward a substrate heated up to $350{ }^{\circ} \mathrm{C}$ under the above-mentioned conditions attains magnetic properties very similar to the ones observed for Gd bulk single crystal: $T_{\mathrm{C}} \sim 293 \mathrm{~K}$ and saturation magnetization of $\sim 2.6 \mathrm{~T}$. Furthermore, they claim to have discovered the reason for detrimental magnetic properties of $\mathrm{Gd}$ films grown at room temperature: smaller grain sizes and larger fraction of fcc Gd paramagnetic (PM) phase and residual strain arising from the growth process. ${ }^{41}$

In parallel, a significant effort has been undergone to produce Gd nanoparticles; however, mostly due to their high reduction potential and highly reactive nature at the nanoscale, this task has proven to be very difficult. Mathew and coworkers successfully tackled this challenge by synthesizing nanocrystalline Gd (grain size around $12 \mathrm{~nm}$ ) through an inert-gas condensation technique using bulk $\mathrm{Gd}$ as the starting material. Its magnetic characterization has unveiled significant irreversibility which was attributed to intra- and interfacial anisotropies which hinder the complete alignment of the magnetic moments (saturation) and consequently significantly diminishes (in comparison to bulk) the magnetic entropy change even for fields as high as $50 \mathrm{kOe}^{45}$ Using a similar method, Aruna and coworkers have produced single Gd particles. To minimize further the oxygen content in the chamber, prior to deposition, a small amount of $\mathrm{Gd}$ was evaporated onto the shutters to serve as getter. The resultant 5-7 $\mathrm{nm}$ diameter nanoparticles have developed a core-shell morphology, with $\mathrm{Gd}_{2} \mathrm{O}_{3}$ and $\mathrm{Gd}-\mathrm{H}$ at the outer and inner nanoparticle regions. More interestingly, they have shown a size-induced structural transformation from the hexagonal (typical of bulk Gd) to a cubic fcc structure in the 5-7 $\mathrm{nm}$ diameter range. ${ }^{46}$ These results are in accordance with the study of Morozov and coworkers developed in 1975, where they have observed the same crystallographic change (from hcp to fcc) in nanoparticles with $\sim 20 \mathrm{~nm}$ diameter, which also lead to the disappearance of any magnetic ordering above $2 \mathrm{~K}^{47}$ The oxidation typically observed in $\mathrm{Gd}$ nanoparticles appears to have been greatly reduced by Ertas and coworkers who have recently developed a novel nanofabrication route based on nanosphere lithography combined with the metal reduction process. This technique resulted in the production of oxide-free Gd nanoparticles, with tunable size and monodisperse nature (up to $5 \%$ ), high magnetization (206 emu/g at $2 \mathrm{~K}$ ), and great potential in magnetic resonance imaging. ${ }^{48}$

Another interesting approach has been followed by Zeng and coworkers who have prepared Gd nanoparticles via an inert-gas condensation method and then have consolidated into a bulk compound through spark plasma sintering in the $250^{\circ}-700{ }^{\circ} \mathrm{C}$ temperature range. ${ }^{49}$ TEM micrographs show different grain sizes in the $15-1000 \mathrm{~nm}$ range, depending in the sintering temperature. Their thorough magnetic characterization has evidenced a similar to bulk second-order magnetic transition although at temperatures slightly different from the bulk part. More dramatically, they have shown that the nanometer-sized grain samples present a $\Delta S_{\mathrm{m}}{ }^{\max } 50 \%$ reduction when compared with micrometer-sized grains. Such reduction is associated with a broadening of the $\Delta S_{\mathrm{m}}(T)$ curve which in turn can be explained by an increasing magnetic and structural disorder in nanocrystalline systems caused by different phenomena such as asmagnetic symmetry breaking, nanoparticles size, and stress distribution-leading to a $T_{\mathrm{C}}$ distribution, as detailed by Amaral and Amaral. ${ }^{50}$ As will be seen throughout this review, the broadening of the $\Delta S_{\mathrm{m}}(T)$ curve is a typical phenomenon in polycrystal micro- and nanoscale systems.

Although Gd nanostructures have received more attention (and are in itself worth of a comprehensive review) than other pure rare-earths, there has been increasing interest in the other elements of this family, triggered by the innovative theoretical studies by Mello and Filho and respective coworkers. ${ }^{51-53}$ In contrast to Gd, Terbium (Tb), Dysprosium (Dy), and Holmium (Ho) present strong anisotropic magnetic properties because of their incomplete $4 f$ orbitals. Dy and Tb are known to have a helical magnetic structure with a period of few unit cells $(<20)$ as a ground state. However, if a very thin film is produced with a thickness lower than this period $(t<$ $15 \mathrm{~nm}$ ), the helical order will be truncated. Furthermore, the spins at the film surface will experience a break in symmetry and will reduce their correlations with second neighbors. ${ }^{53}$ These theoretical studies have shown that in 
such circumstances, the pure ferromagnetic (FM) ordering will be favored by these spins and ultimately, by applying a magnetic field strong enough $(>0.6 \mathrm{kOe})$, one is able to completely suppress the helical magnetic state in favor of a collinear FM one. Obviously, such transformation will lead to an enhanced overall magnetic moment, as can be seen in the magnetization versus temperature, $M(T)$ curves, shown in Fig. 2. One of the many interesting consequences arising from this transformation is the enhancement of the Dy and Tb MCE in comparison with their bulk counterpart.

$\mathrm{R}=\mathrm{Tb}$ and Dy thicker $(100 \mathrm{~nm})$ films deposited by DC sputtering onto an oxidized and preheated $\left(350{ }^{\circ} \mathrm{C}\right.$ or room temperature) $\mathrm{Si}$ substrate have shown magnetic transition temperatures slightly lower, as Scheunert and coworkers have shown. ${ }^{43}$ Surprisingly, they have also found the suppression of the antiferromagnetic (AFM) helical magnetic state for such thicker films, resulting in a direct transition from PM to FM state for both the Dy and the $\mathrm{Tb}$ thin films, which they say is not a consequence of the surface spins, but rather a result of the lattice expansion caused by strain in these films.

The size-reduction of rare earth alloys (with other rare earths or metals) was studied by Shao and coworkers when they studied $\mathrm{Gd}-\mathrm{Tb}, \mathrm{Gd}-\mathrm{Y}$, and $\mathrm{Gd}-\mathrm{Zn}$ nanocomposite ribbons. ${ }^{54}$ In this work, the authors have performed a rapid-quenching $\left(\sim 10^{5} \mathrm{~K} / \mathrm{s}\right)$ on a meltspinner of the arc-melted bulk alloys. Afterward, the resulting amorphous particles with $\sim 20 \mathrm{~nm}$ diameter were immersed in acetone and milled for $125 \mathrm{rpm}$ for $100 \mathrm{~h}$. This process induced the agglomeration and recrystallization. Interestingly, they found that the final powder, with diameter $<360 \mathrm{~nm}$, exhibited a higher specific heat, but lower $T_{\mathrm{C}}$ and MCE in the near roomtemperature range in comparison with the respective bulk.

Concerning other single elements, besides rare-earths, leads Franco and coworkers to explore $\mathrm{Ni}$ nanowire magnetic properties. ${ }^{55}$ In fact, they initially predicted a major change in the magnetic properties of this nanostructure, promoted by the enhanced shape anisotropy in nanowires and the consequent strong magnetic anisotropy. They demonstrated experimentally and by modeling the coexistence of positive (inverse) and negative (normal) MCE in these nanowires and they found that such positive/negative nature could be tuned according to the applied field value.

\section{B. $\mathbf{R}_{\mathbf{5}}(\mathbf{S i}, \mathbf{G e})_{4}$}

The $\mathrm{R}_{5}\left(\mathrm{Si}_{x} \mathrm{Ge}_{1-x}\right)_{4}(\mathrm{R}=$ rare earth element $)$ family of compounds is a fruitful example of a strongly coupled system. In 1997, a study by Pecharsky and Gschneidner leads to the rejuvenation of the magnetic refrigeration research at room temperature-as is verified by its more than 2000 citations nowadays. ${ }^{2}$ The Ames group reported a giant MCE of $\sim 19 \mathrm{~J} /(\mathrm{kg} \mathrm{K}) / 15 \mathrm{~K}$ of $\Delta S_{\text {iso }} / \Delta T_{\text {ad }}$ under a $50 \mathrm{kOe}$ applied field for the $\mathrm{Gd}_{5} \mathrm{Si}_{2} \mathrm{Ge}_{2}$ compound at $\sim 290 \mathrm{~K}$. Shortly after, Morellon and coworkers ${ }^{7}$ found that such an effect was due to the strong coupling between magnetic and lattice orders of freedom, which resulted in the so-called magnetostructural transition. Attracting immediate materials scientist's attention, different $\mathrm{R}_{5}\left(\mathrm{Si}_{x} \mathrm{Ge}_{1-x}\right)_{4}(\mathrm{R}=$ rare earth $)$ families were thoroughly investigated. Their complete phase diagrams
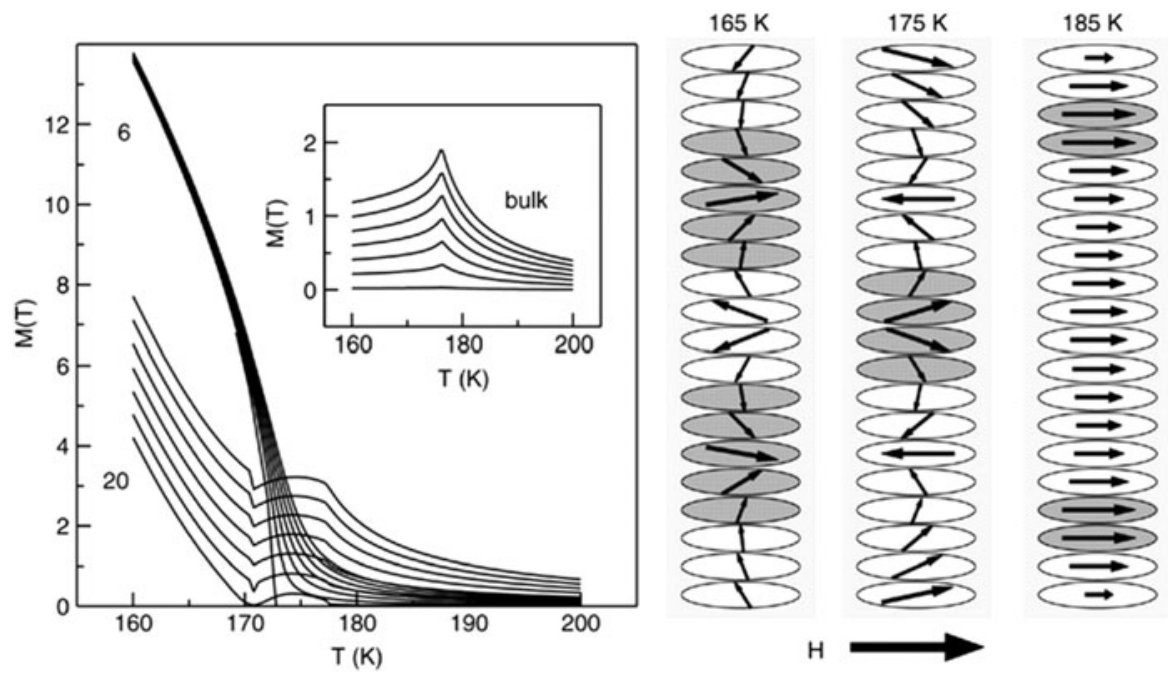

FIG. 2. Seven isofield magnetization curves as a function of temperature (in the field range $[0,0.6] \mathrm{kOe}$ ) for a thinner (six atomic layers) and a thicker (twenty atomic layers) Dy thin film. The inset shows the same seven isofield magnetization curves but for Dy bulk material. The helicoidally spin structure in the twenty unit cells of the film for three selected temperatures is shown schematically at the right side for a constant $H=0.6$ kOe. Reprinted with permission from Ref. 53 . 
were described, revealing a complex set of phase regions and phase transitions that were classified by Miller as a "playground for material scientists" 35 and that can be resumed into three major atomic structures and three major magnetic orders: orthorhombic $\mathrm{O}(\mathrm{I})$, orthorhombic $\mathrm{O}(\mathrm{II})$, and monoclinic (M) atomic structures and PM, FM, and AFM magnetic orders. ${ }^{56}$ It was also found that the GMCE was not the only consequence of the magnetostructural transition but that it also promotes giant magnetoresistive $^{57}$ and colossal magnetostriction effects. ${ }^{58}$ Due to all these reasons, it is surprising to observe the few amounts of studies dedicated to the study of these materials scale reduction. Nevertheless, there are a handful of interesting reports, namely, by Moore and coworkers at Imperial College, which showed for the first time that fragmenting a $\mathrm{Gd}_{5} \mathrm{Ge}_{4}$ bulk sample in small pieces, $\sim 100 \mu \mathrm{m}$, leads to a $20 \%$ reduction of the critical magnetic field required to complete the corresponding phase transition in comparison with the bulk counterpart. ${ }^{25}$ They attributed this effect to the reduction of magnetic domains per particle (approximately 3-5 domains) that implies a reduction/removal of strain fields within neighboring magnetic domains. Trevizoli and coworkers have also studied the effects of microstructuring $\mathrm{Gd}_{5.09} \mathrm{Ge}_{2.03} \mathrm{Si}_{1.88}$, via metallurgical pulverization. They found that for samples with lower particle sizes $(25<d<53 \mu \mathrm{m})$, the first-order transition is suppressed during the sintering process, whereas for larger particles, the giant MCE is retained. ${ }^{59}$ One of the most popular approaches for down-sizing magnetocaloric materials has been ball-milling as Blasquez and coworkers have reviewed, ${ }^{60}$ and this technique has been intensively used in particular for the $\mathrm{R}_{5}(\mathrm{Si}, \mathrm{Ge})_{4}$ materials. ${ }^{61-63}$ It was observed that for materials with first-order magnetostructural transitions, the ball-milling and consequent sizereduction (downwards to the $0.5-5 \mu \mathrm{m}$ range) lead to a strong attenuation of the respective MCE, which is explained by the increasing amorphization with increasing milling time. However, our group unveiled the mechanism that is ruling the different behaviors: it is an induce internal strain arising from the ball-milling process during the formation of microparticles. ${ }^{61}$ We further showed that this effect, depending on the exact composition of the $\mathrm{R}_{5}(\mathrm{Si}, \mathrm{Ge})_{4}$ family, could lead to a $23 \%$ MCE enhancement of the $\mathrm{Tb}_{5} \mathrm{Si}_{2} \mathrm{Ge}_{2}$ and, by contrast, to a $35 \%$ MCE decrease for $\mathrm{Gd}_{5} \mathrm{Si}_{1.3} \mathrm{Ge}_{2.7}$ milled particles (1-10 $\mu \mathrm{m})$.

In 2005, Sambandam and coworkers ${ }^{64}$ reported their attempt to produce thin films by sputtering with a DC/ RF-magnetron sputtering system, a $\mathrm{Gd}_{5} \mathrm{Si}_{2} \mathrm{Ge}_{2}$ sputter target that was produced by cold-pressing and sintering GdSiGe powder into a $76 \mathrm{~mm}$ disc. However, they discovered that the buffer layer used, silicon nitride $\left(\mathrm{Si}_{3} \mathrm{~N}_{4}\right)$, was not effective in preventing $\mathrm{Si}$ diffusion from the $\mathrm{Si}$ substrate and consequently no major evidence of
$\mathrm{Gd}_{5}(\mathrm{Si}, \mathrm{Ge})_{4}$ phase formation was detected. In 2013, Hadimani and coworkers ${ }^{65}$ were able to deposit a Gd$\mathrm{Si}-\mathrm{Ge}$ thin film by using a femtosecond pulsed laser to ablate a $\mathrm{Gd}_{5} \mathrm{Si}_{2.09} \mathrm{Ge}_{1.91}$ target onto a $200{ }^{\circ} \mathrm{C}$ heated $\mathrm{Si}$ substrate. Due to its high energy pulse, the material is sputtered from the target in large amounts/grains, which then land on the substrate surface, retaining its target composition. However, the X-ray diffraction (XRD) analysis performed by the authors revealed also the presence of other phases, such as $\mathrm{Gd}_{5} \mathrm{Si}_{4}$ and $\mathrm{Gd}_{5} \mathrm{Ge}_{3}$. Its magnetic characterization shows a small transition above the $298 \mathrm{~K}$, but no evidence of a first-order transition. Later, our team used the same deposition method to deposit a $\mathrm{Gd}_{5} \mathrm{Si}_{1.3} \mathrm{Ge}_{2.7}$ target resulting in an ensemble of spherical nanogranular particles with $\sim 80 \mathrm{~nm}$ mean size and total thickness $\sim 780 \mathrm{~nm} .{ }^{66}$ The nanogranular film presented an incomplete structural transition at $\sim 190 \mathrm{~K}$ coupled with a FM $\rightarrow$ PM magnetic transition, resulting in: (i) a colossal anisotropic lattice parameter change, $\Delta a / a \sim 1.2 \%$, as can be seen in Fig. 3(a); (ii) a relatively large $-\Delta S_{\mathrm{mag}} \sim 8.9 \mathrm{~J} /(\mathrm{kg} \mathrm{K})$ (at $\Delta H=50 \mathrm{kOe}$ ), that however represents only $33 \%$ of the counterpart bulk value. In accordance with the ballmilling results, here too, the low-volume $\mathrm{O}(\mathrm{I})$ phase is favored. The major magnetic and structural changes observed in the film in comparison with the bulk form were attributed to the nanoparticles' intrinsic pressure, estimated in 8-11 kbar.

Further studies demonstrated that this thin film presents low-resistance to thermal cycles, leading to a suppression of its magnetostructural transition when the number of cycles increases beyond $1000 .{ }^{67}$ Similar to the bulk behavior, it is also possible to tune the thin film Curie temperature and its preferred atomic structure by post-heat treatments, although in contrast with the high-temperatures needed for the bulk counterparts $\left(1000-1300{ }^{\circ} \mathrm{C}\right)$, in the thin film smaller temperatures are required $\left(400-600{ }^{\circ} \mathrm{C}\right) .{ }^{68}$

\section{FeRh}

Iron-rhodium (FeRh) is a binary system, which became well known at its bulk form due to its positive GMCE. In fact, it presents one of the highest refrigerant capacities at room temperature, surpassing the popular $\mathrm{Gd}_{5} \mathrm{Si}_{2} \mathrm{Ge}_{1.9} \mathrm{~F}_{0.1}$ and $\mathrm{MnFeP}_{0.45} \mathrm{As}_{0.55}$ negative GMCE. The bulk FeRh presents a first-order transition resultant of the coupling between the magnetic and the structural transitions; however, its MCE is not fully reversible under laboratorial applied fields. Hence, the expectation of producing it in the thin film form was obviously large. In fact, FeRh films were first grown in the 1966, when their magnetic and electrical transport measurements were first performed. Lommel, ${ }^{69}$ to surpass the significantly different vapor pressures of $\mathrm{Fe}$ and $\mathrm{Rh}$, adopted 

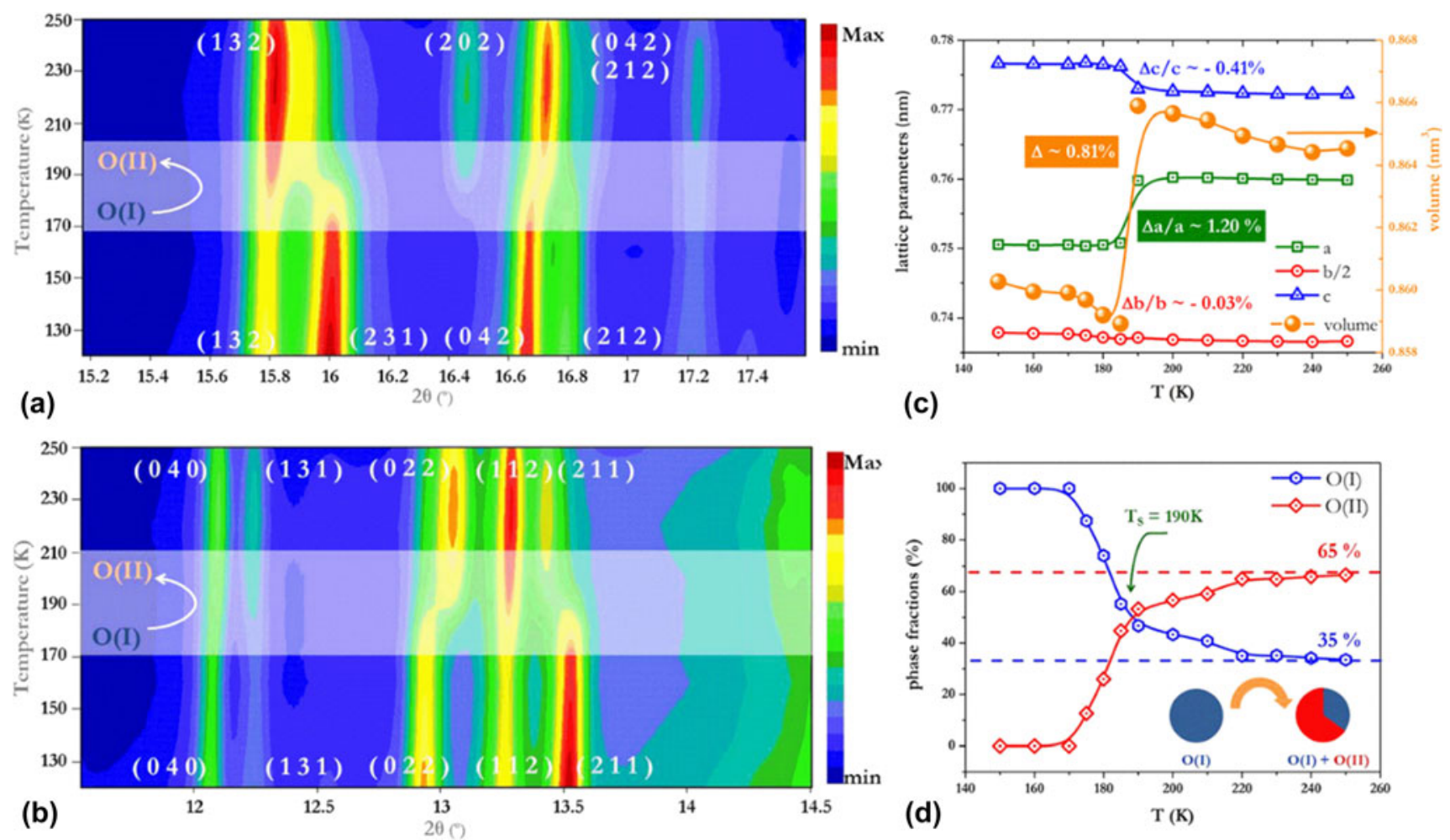

FIG. 3. 2D Contour plot of the collected and analyzed synchrotron X-ray diffracted spectra as a function of temperature ([120, 250] $K$ range) in the $\left[15^{\circ} ; 17.6^{\circ}\right]$ (a) and $\left[11.5^{\circ} ; 14.5^{\circ}\right]$ (b) interval. Temperature dependence of the two phase fractions (d) and the majority phase lattice parameters and volume, assigned to the left and right y-axis, respectively (c). Reproduced with permission from Ref. 66. Copyright 2015 AIP Publishing LLC.

a smart strategy, which consisted in depositing a multilayer system composed by Fe and Rh layers alternately grown by electron beam melting on a glass substrate that was held at $300{ }^{\circ} \mathrm{C}$ during the deposition. As to promote interlayer diffusion and crystallization of the 1:1 phase, Lommel performed an annealing treatment at $565^{\circ} \mathrm{C}$ for $5 \mathrm{~h}$. This author also tried two different strategies: (i) direct co-deposition of $\mathrm{Fe}$ and $\mathrm{Rh}$ by electron beam melting; (ii) by sputtering a $\mathrm{Fe}_{0.50} \mathrm{Rh}_{0.545}$ target. ${ }^{69}$ However, only the alternate approach successfully synthesized the FeRh phase. Both the electrical transport and magnetic measurements evidenced the presence of the firstorder transition, despite it being broader (about $200 \mathrm{~K}$ ) in comparison with the $(\sim 15 \mathrm{~K})$ observed in bulk, as can be seen in Fig. 4(a). Also, the thin film overall magnetic moment was about one third of the bulk one.

It was 50 years later that Zhou and coworkers ${ }^{70}$ published their work on FeRh films, but making use of nowadays modern technology-a DC magnetron sputtering under high vacuum conditions. They have epitaxially grown FeRh $100 \mathrm{~nm}$ thin films on $\mathrm{MgO}\left(\begin{array}{lll}0 & 0 & 1\end{array}\right)$ single crystals heated up to $400{ }^{\circ} \mathrm{C}$ at $4 \times 10^{-3}$ mbar argon working pressure. XRD analysis as a function of temperature unveiled a giant lattice parameter change of $\sim 0.66 \%$ (almost twice the value obtained for the bulk) across the transition temperature $(\sim 325 \mathrm{~K})$, which they attributed to a distortion of the tetragonal structure due to the growth relationship between the film and the substrate. Magnetic measurements have also shown thermal and magnetic hysteresis [in $M(T)$ and $M(H)$ curves, respectively], confirming the first-order nature of this transition. Such an effect resulted in a GMCE, in fact the highest intrinsic effect measured in thin films so far: $\Delta S_{\mathrm{m}}{ }^{\max } \sim 20 \mathrm{~J} /(\mathrm{kg} \mathrm{K})$ for a $\Delta H=50 \mathrm{kOe}$, as can be seen in Fig. 4(b). ${ }^{70}$

Recently, important breakthroughs were achieved to solve the major drawback of the FeRh system, namely, the irreversibility of the effect. First, Cherifi and coworkers explored the voltage control of magnetization in a system composed by a FeRh epitaxially grown on top of $\mathrm{a} \mathrm{BaTiO}_{3}$ substrate. They have demonstrated how a low electric field was able to induce an AFM-FM magnetic transition on the FeRh film via voltage-induced strains in $\mathrm{BaTiO}_{3}{ }^{71}$ Later, they extended the study of the effects of electric voltage application on this system and found that the large hysteresis typically observed in this system can be transferred into an elastic cycle. It is important to stress that this strain mediated effect does not rely on the presence/absence of any structural transition in the $\mathrm{BaTiO}_{3}$ substrate, in contrast with recent 

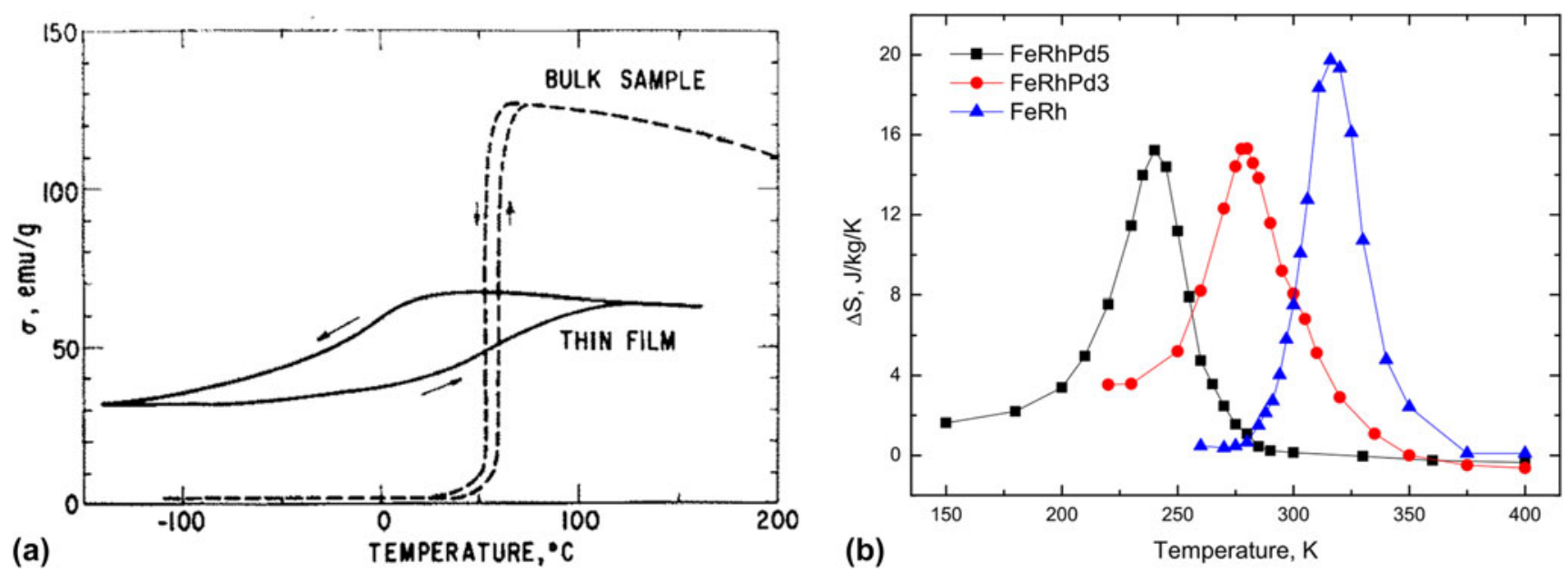

FIG. 4. (a) Magnetization curves as a function of temperature for a FeRh bulk and thin film sample. (b) Magnetic entropy change of $\mathrm{FeRh}, \mathrm{FeRhPd}$, and $\mathrm{FeRhPd}_{5}$ thin films as a function of temperature. Reproduced with permission from (a) Ref. 69 and (b) Ref. 70.

studies. ${ }^{72}$ Therefore, Liu and coworkers suggest a smartmulticaloric refrigeration cycle that could take advantage of this hysteresis suppression via a dual-stimulus (magnetic and electric) approach. In parallel, FeRh mesoscale stripes have also been attracting significant attention since this mesoscale is the same as the typical crystallographic grains present in bulk FeRh and hence its study allows to inspect the FeRh phase transition within its heterogeneity range. Uhlir and coworkers have grown epitaxial FeRh thin films with $25 \mathrm{~nm}$ thickness on top of the $\mathrm{MgO}$ substrate and later have patterned it into stripes with different widths, such as 550 and $1100 \mathrm{~nm}$. They have unveiled a significantly larger (orders of magnitude) asymmetry in the electrical resistivity heating and cooling curves on the $550 \mathrm{~nm}$ width stripes in comparison with typical wider thin films, which they ascribed to the rapid nucleation of the AFM region throughout the stripes, whereas in thin films, there are much more nucleation sites and the nucleation occurs along a wider temperature region. ${ }^{73}$ In contrast with thin films, stripes and larger nanoparticles, Hillion and coworkers found that small $(<5 \mathrm{~nm})$ nanoparticles retained the high-temperature FM state until low temperatures (down to $3 \mathrm{~K}$ ) suggesting a rich size-dependent phase diagram for the FeRh system. $^{74}$

As numerous examples illustrate, the FeRh system is probably the magnetocaloric system that has profited the most from the size-reduction route, unveiling promising technological advantages (such as the reduction of the magnetic irreversibility) and interesting new physical states. In the near future, more detailed nanoparticles size-dependent studies should help understand what are the main mechanisms driving the magnetic ordering at this scale.

\section{MnAs}

The binary bulk MnAs compound was first synthesized in the $1950 \mathrm{~s}$ and its adiabatic temperature change $\Delta T^{\text {ad }}$ was measured for the first time in 1980 by Kuhrt and coworkers, ${ }^{75}$ delivering a rather small value of $\sim 0.2 \mathrm{~K}$ for a $\Delta H=6.5 \mathrm{kOe}$. Nevertheless, this initial measurement did not convince Wada and Tanabe, ${ }^{11}$ who decided, after the advent of the Pecharsky and Gschneidner discover, ${ }^{2}$ to remeasure the MnAs magnetocaloric properties and surprisingly they measured a giant magnetocaloric response: $-\Delta S^{\max } \sim 40 \mathrm{~J} /(\mathrm{K} \mathrm{kg})$ for a $\Delta H^{\text {ad }}=50$ kOe and obtained a $\Delta T^{\text {ad }} \sim 2.5 \mathrm{~K}$ for $\Delta H^{\text {ad }}=10 \mathrm{kOe}$ (almost ten times the $\Delta T^{\mathrm{ad}} / \Delta H^{\mathrm{ad}}$ ratio obtained by Kuhrt). ${ }^{75}$ It was found that in the MnAs-based materials, the MCE is associated with a complex magnetostructural phase transition. The $\alpha$-MnAs phase crystallizes in a FM hexagonal NiAs-type structure, which is stable up to 318 $\mathrm{K}$, when it transforms into orthorhombic $\beta$-MnAs by a first-order phase transition. ${ }^{76}$ In 2004, Gama and coworkers $^{77}$ had reported a MnAs colossal entropy change $[267 \mathrm{~J} /(\mathrm{K} \mathrm{kg})]$ induced both by pressure and magnetic field, which was later interpreted as the impossibility of using Maxwell state equation at first order transitions, resulting then in the appearance of MCE measurements standards to suppress these limitations. ${ }^{78,79}$

At the nanoscale, in 2008 Mosca and coworkers, ${ }^{80}$ acknowledging the fact that MnAs FM interactions are strongly anisotropic and susceptible to subtle structural changes, decided to grow epitaxially MnAs films on top of two gallium arsenide (GaAs) substrates with different orientations [ $\left[\begin{array}{lll}0 & 0 & 1\end{array}\right)$ and $\left.\left(\begin{array}{lll}1 & 1 & 1\end{array}\right)\right]$. They grew MnAs $70 \mathrm{~nm}$ epilayers by molecular beam epitaxy on heat-treated $\left(260{ }^{\circ} \mathrm{C}\right)$ substrates. To prevent oxidation and achieve 
optimal surface quality, the substrates were heat treated at $580{ }^{\circ} \mathrm{C}$ under As flux and then were deposited with a GaAs buffer layer. The complete process was monitored by reflection high-energy electron diffraction diagrams, which together with XRD allowed to ensure the desired epitaxial relations. Due to the large mismatch between the substrate and film unit cells, the $\left(\begin{array}{lll}1 & 1 & 1\end{array}\right)$ substrate induces a strong $(0.82 \%)$ compressive strain in the basal plane leading to a premature appearance of the $\beta$ phase and consequently to a broadening of the magnetostructural transition, as is clearly observed in its magnetization as a function of temperature curves. ${ }^{80}$ This did not happen in the $\left(\begin{array}{lll}1 & 0 & 0\end{array}\right)$ case due to the different configuration of the unit cells, and hence its transition occurs in a narrower temperature interval. Kaganer and coworkers $^{81}$ had already demonstrated this wider phase coexistence, although they did not present the complementary magnetic study. As expected, such constraining reflects on the MCE of both cases leading to a sharper and higher MCE under a $\Delta H=50 \mathrm{kOe}$ for the $\left(\begin{array}{lll}1 & 0 & 0\end{array}\right)$ case: $\sim 20 \mathrm{~J} /(\mathrm{K} \mathrm{kg})$ in comparison with $\sim 7 \mathrm{~J} /(\mathrm{K} \mathrm{kg})$ for the $\left(\begin{array}{lll}1 & 1 & 1\end{array}\right)$ case, as can be seen in Fig. 5. These results were in good agreement with simple thermodynamic model considering a homogeneous strain across the MnAs epilayers and underlined the importance of MnAs compatibility with worldwide used $\mathrm{Si}\left(\begin{array}{lll}0 & 0 & 1\end{array}\right)$ and GaAs semiconductors concerning the potential development of micromagnetic-refrigerators.

In 2014, Trassineli and coworkers ${ }^{82}$ presented an alternative to reduce the thermal hysteresis in these MnAs/GaAs systems: a highly charged ion bombardment. Magnetic force microscopy images clearly show the increasing number of random defects as the ion fluence increases. Furthermore, such defects facilitate

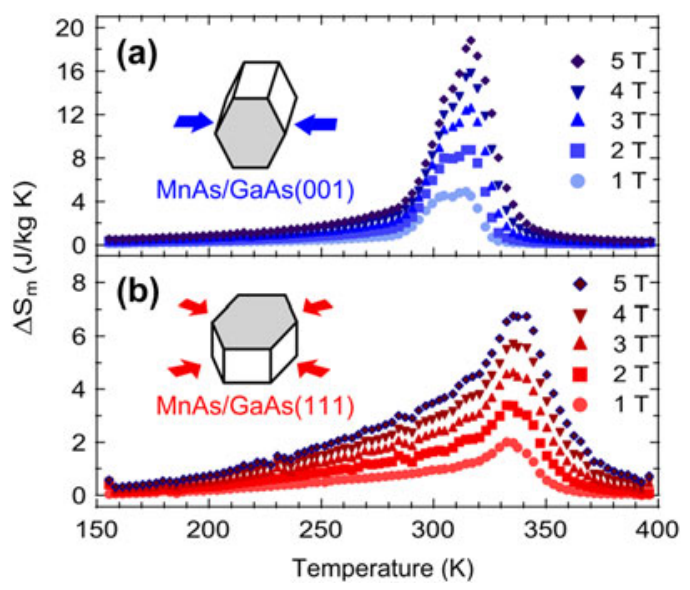

FIG. 5. Magnetic entropy changes as a function of temperature and field for (a) MnAs/GaAs ( $\left.\begin{array}{llll}0 & 0 & 1\end{array}\right)$ and (b) MnAs/GaAs ( $\left.\begin{array}{lll}1 & 1 & 1\end{array}\right)$ epilayers. Magnetic field was applied in the direction of the easy magnetic axis of MnAs epilayers. Reprinted figure with permission from Ref. 80 . the nucleation of one phase with respect to the other in the magnetostructural transition, which consequently accelerates the phase transition and leads to a decrease of its thermal hysteresis. Mosca and coworkers ${ }^{80}$ certainly paved the way for other interesting possibilities on MnAs/GaAs structures. One example is that of Duquesne and coworkers, ${ }^{83}$ whom have deposited a multilayer system: $1 \mu \mathrm{m}$ zinc oxide $(\mathrm{ZnO})$ piezoelectric layer deposited on the top of a gold buffer layer $(300 \mathrm{~nm})$, which was on top of the epitaxially grown MnAs epilayer with $100 \mathrm{~nm}$ on top of a GaAs substrate. By emitting an acoustic surface wave triggered by using an electrical emitter patterned on the $\mathrm{ZnO}$ piezoelectric layer in an interval range around room temperature $\left(0-60{ }^{\circ} \mathrm{C}\right)$, the authors observed that ultrasonic waves $(170 \mathrm{MHz})$ are strongly attenuated by the MnAs phase coexistence (up to $150 \mathrm{~dB} / \mathrm{cm}$ ). They showed that such attenuation was caused by the MnAs MCE and hence they demonstrated the two-way relationship between mechanical control and magnetic properties and their (almost) endless opportunities. Already in 2014, another interesting idea arose from Spezzani and coworkers, ${ }^{84}$ as they were able to reverse $\mathrm{Fe}$ magnetization on a $\mathrm{Fe} / \mathrm{MnAs} / \mathrm{GaAs}$ multilayer structure through the action of a 100-femtosecond optical pulse (used to trigger local thermal variations) and a 100femtosecond X-ray pulse to probe the magnetic and structural dynamics of such multilayer. They were able to observe both the Fe magnetization reversal and the MnAs structural transition, despite the time interval between them was only of a few picoseconds. ${ }^{84}$

\section{E. $\mathrm{La}(\mathrm{Fe}, \mathrm{Si})_{13}$ family}

$\mathrm{The} \mathrm{La}(\mathrm{Fe}, \mathrm{Si})_{13}$ family of materials is, together with $\mathrm{Mn}-\mathrm{Fe}-\mathrm{P}-\mathrm{Si}$, one of the most promising magnetocaloric materials to be used in room temperature magnetic refrigeration applications. They have been thoroughly studied in the past 10 years; however, surprisingly, very few studies dedicated to their micro- and nanostructuring can be found. One of the first attempts to change the microstructure of $\mathrm{La}-\mathrm{Fe}-\mathrm{Si}$ was ingeniously developed by Lyubina and coworkers when they introduced up to $30 \%$ of porosity in a bulk $\mathrm{La}-\mathrm{Fe}-\mathrm{Si}$ sample by crushing an arc-melted button and further press the resulting powder under vacuum using a hot-press setup. Increasing the density of pores in the final pellets resulted in: a reduction of thermal hysteresis (from $2.3 \mathrm{~K}$ in the bulk down to below $0.5 \mathrm{~K}$ in the porous material), a great improvement of mechanical stability (bulk counterpart loses mechanical integrity after less than 10 cycles, in contrast with the porous which remains stable even after more than 600 cycles), and a $20 \%$ decrease in $\Delta T^{\text {ad }}$. These results were interpreted as a consequence of the removal of the internal constraints, such as the grain boundaries, in the porous material. ${ }^{85}$ Lyubina extended 
this study on porous materials down to nanometer-sized crystallites shortly after, ${ }^{86}$ where it was observed: an advantageous attenuation of the magnetic hysteresis, but a detrimental strong (40/60\%) reduction of the $\Delta S^{\max }$ for $70 / 44 \mathrm{~nm}$ sized crystallites, respectively. In conclusion, the authors proposed that an optimal magnetocaloric compound should be comprised off: micro- and single crystalline particles with a uniform size distribution to sharpen the magnetic transition and consequently enhance its MCE. Also for this family, most of the sizereduction studies were performed through up-bottom approach, by using ball-milling process, ${ }^{87-90}$ or simply hand grinded particles. ${ }^{88,91,92} \mathrm{Hu}$ and coworkers have found that the hysteresis loss reduces as the particle size is decreased and such reduction can be as high as $61 \%$ for particles within the $20-50 \mu \mathrm{m}$ range. Furthermore, they have narrowed an optimal size interval, 20-120 $\mu \mathrm{m}$, where the hysteresis loss is greatly reduced but the large entropy change is retained. ${ }^{89}$ They have attributed this reduction to the reduction of the so-called extrinsic hysteresis, associated with the heat transfer kinetics, as also identified by Gutfleisch and coworkers. ${ }^{93}$ In particular, $\mathrm{Hu}$ and coworkers estimate that there is a 27-time reduction of the surface area versus volume ratio while decreasing from the bulk-like particles $(>100 \mu \mathrm{m})$ toward smaller size $(\sim 20 \mu \mathrm{m})$, which will greatly increase the thermal exchanges between the particles and the surrounding. ${ }^{89}$ This point is particularly important considering the fact that, due to its poor mechanical properties, $\mathrm{La}-\mathrm{Fe}-\mathrm{Si}$ is planned to be immersed in a polymer/metal matrix to form a composite-the magnetic refrigerant to be used in a real magnetic refrigeration device. Consequently, the smaller the magnetocaloric particles are, the denser and the harder will be the final composite material. In fact, several studies have been published during the last years devoted to the optimal construction of such a composite. ${ }^{23,94,95}$ Interestingly, due to the above-mentioned dramatic increase of the surface area versus volume ratio, the particle-size reduction has also proven to be useful during the hydrogenation of $\mathrm{LaFeSi}$ compounds: enabling an homogeneous distribution, a greater mechanical stability, and a large magnetic entropy change, as shown by Zhang and coworkers. ${ }^{92}$ While further decreasing the size toward the nanoscale, an important theoretical study performed by Zhang and coworkers deserves highlight. They have applied Landau-Ginzburg expansion of the free energy density and the Arrhenius-Néel statistical switching model to simulate the magnetization behavior within the metamagnetic transition temperature region and have found that the smallest nucleation site possible for a new phase to emerge is about $4.2 \mathrm{~nm}$ : i.e., this is the critical size below which no metamagnetic transition is expected. This year a report was published dedicated to the $\mathrm{LaFeSi}$ nanostructure produced by spark ablation of
LaFeSi bulk rods. ${ }^{96}$ In this attempt, the $6 \mathrm{~nm}$ (mean size) produced $\mathrm{LaFeSi}$ nanoparticles exhibited a much broader magnetic transition than the bulk counterpart in accordance with the magnetic behavior exhibited by NPs of other magnetocaloric materials. Moreover, they have demonstrated that the NPs' Curie temperature can be tuned by controlling ablation parameters toward room temperature. This work is expected to open for once the pathway toward nanoscale for LaFeSi compounds, enabling to explore size-reduction effects and confirm the existence of a critical size for the presence of the metamagnetic transition.

\section{F. $\mathrm{Fe}_{2} \mathrm{P}$ family}

The $(\mathrm{Mn}-\mathrm{Fe})_{2}(\mathrm{P}, \mathrm{As}, \mathrm{Si}, \mathrm{B})$ materials derive from iron phosphide- $\mathrm{Fe}_{2} \mathrm{P}$ and together with the $\mathrm{La}-\mathrm{Fe}-\mathrm{Si}$ family, are the most promising magnetocaloric materials for room temperature magnetic refrigeration applications. Tegus and coworkers have shown first that these materials stabilize in the hexagonal $\mathrm{Fe}_{2} \mathrm{P}$ structure and undergo sharp magnetic transition at room temperature, leading to a giant MCE, with a $\Delta S^{\max }$ twice the one observed for pure gadolinium and similar to the one exhibited by $\mathrm{Gd}_{5} \mathrm{Si}_{2} \mathrm{Ge}_{2} \cdot{ }^{97}$ Later, Dung and coworkers demonstrated that the $T_{\mathrm{C}}$ of this family could be tuned in a wide temperature interval, $200-400 \mathrm{~K}$, by adjusting the metal to nonmetal ratio and by chemical substitution, in particular with boron. ${ }^{98,99}$ Despite its leading role in the magnetocaloric race at bulk scale, there are few reports dedicated to the scale reduction effect on its magnetic properties. However, the first attempts toward this goal were developed already in 1995, when Lukehart and coworkers have shown that a covalent incorporation of an organoiron complex into a silica xerogel matrix resulted in a single-source for two different nanocomposites: $\mathrm{Fe}_{2} \mathrm{P}$ and $\mathrm{Fe}_{2} \mathrm{O}_{3}$. In this work, they demonstrated that thermal treatments under hydrogen atmosphere promoted the production of an ensemble of nanocrystals, including $\mathrm{Fe}_{2} \mathrm{P}$ hexagonal-like nanocrystals with $\sim 4.7 \mathrm{~nm}$ typical size. Their incomplete magnetic characterization unveiled a super PM behavior at room temperature, whereas at $10 \mathrm{~K}$, a typical mixture of FM and PM components was found. ${ }^{100}$ Almost ten years later, Park and co-authors have published a report on the first $1 \mathrm{D} \mathrm{Fe}_{2} \mathrm{P}$ systems produced by a novel synthesis method comprising thermal decomposition of continuously supplied iron pentacarbonyl in trioctylphosphane using a syringe pump. By varying the injection rate, they were able to tailor the $\mathrm{Fe}_{2} \mathrm{P}$ nanorod lengths $(88,107$, and $209 \mathrm{~nm})$ while retaining similar width $(5-6 \mathrm{~nm})$. More interestingly, they have shown that these nanorods self-assemble in a long-range order 3D hexagonal super-structure. Their atomic structure is hexagonal, like in bulk counterpart; however, their magnetic behavior depends on their 
lengths, in particular, their blocking temperature increases with the length size, up to $\sim 250 \mathrm{~K}^{101} \mathrm{In}$ addition, $\mathrm{Fe}_{2} \mathrm{P}$ nanoparticle research triggered another application for these transition metal alloys as catalysts for photocatalytic hydrogen evolution. ${ }^{102-103}$ Several studies demonstrate the low-cost $\mathrm{Fe}_{2} \mathrm{P}$ nanoparticles formation, their enhanced surface area, excellent electrochemical properties, and mechanical stability after thousands of cycles. The same motivation led Schipper and coworkers to grow $\mathrm{FeP}, \mathrm{Fe}_{2} \mathrm{P}$, and $\mathrm{Fe}_{3} \mathrm{P}$ thin films recently. ${ }^{104}$ The films were grown by metal-organic chemical vapor deposition using single-source molecular precursors $\left(\mathrm{Fe}(\mathrm{CO})_{4} \mathrm{PH}_{3}\right)$ on fluorine-doped tin oxide to evaluate the system performance in hydrogen evolution. Both the experimental and the density functional theory calculations results show a higher hydrogen coverage for Fe-rich nanoparticles in comparison with P-rich. Following the same procedure, the same group of authors produced the $\mathrm{Mn}$ substituted, $(\mathrm{MnFe})_{2} \mathrm{P}, 1-3 \mu \mathrm{m}$ thick films on top of alumina and quartz substrates. They have found a nanogranular-like morphology with triangular and spherical shapes for quartz and alumina substrates, whereas both exhibit the typical $\mathrm{Fe}_{2} \mathrm{P}$ hexagonal crystal structure. Both films exhibit a FM and superparamagnetic nature due to their small crystallite sizes. ${ }^{105}$ It is important to remark the stability of the $\mathrm{Fe}_{2} \mathrm{P}$ hexagonal phase in all the above-mentioned nanostructures in contrast with the metastable nature of this phase in the bulk compounds, typically requiring a long series of high temperature thermal treatments. Unfortunately, there are no published reports completely dedicated to the influence of size-reduction on the magnetocaloric properties of $\mathrm{Fe}_{2} \mathrm{P}$ or more generally $(\mathrm{Mn}, \mathrm{Fe})_{2}(\mathrm{P}, \mathrm{Si})$. Due to the increasing relevance, this material has been receiving at the macroscale, and its size-reduction studies are certainly an open avenue yet to explore.

\section{G. FM shape memory alloys}

Ferromagnetic shape memory alloys (FSMAs) or magnetic shape memory alloys (MSMAs) are terms technically attributed to the materials that have the ability to show deformations when subjected to magnetic fields and that present two (typically, austenite and martensite) or more crystallographic phases for which reversible transitions from one to the other occur through diffusion-less transformations. ${ }^{106}$ The MSMAs were discovered in 1996 when the $\mathrm{Ni}_{2} \mathrm{MnGa}^{107}$ was first studied. A few years later, $\mathrm{Hu}$ et al. ${ }^{10}$ unveiled that $\mathrm{Ni}_{51.5} \mathrm{Mn}_{22.7} \mathrm{Ga}_{25.8}$ displays a large MCE due to the martensitic to austenitic structural transition. So, basically from the interplay between martensitic transformations and magnetism, two important mechanisms are identifiable: (i) magnetic field induced martensitic transformation (magnetic field dependence of the martensitic transformation temperature) and (ii) the magnetically induced reorientation of variants in the martensitic phase (high magnetocrystalline anisotropy and low elastic shear modulus of martensite that enables twin boundary displacement in response to the application of a magnetic field). ${ }^{108,109}$

Off the FSMA, we highlight the Heusler alloys-the most widely studied materials. Two families of Heusler alloys are reported: (i) half Heusler alloys with chemical composition XYZ and (ii) full Heusler alloys that are represented by the chemical composition: $\mathrm{X}_{2} \mathrm{YZ}$ ( $\mathrm{X}$ and $\mathrm{Y}$ are transition metals or lanthanides, rare-earth elements, and $\mathrm{Z}$ a main group metal or a semimetal). $\mathrm{Ni}_{2} \mathrm{MnGa}$ belongs to this last category and stabilizes in the $\mathrm{L} 2_{1}$ crystal structure at room temperature exhibiting the martensitic transformation for this exact stoichiometry ${ }^{110}$; whereas, the Ni-Mn-X (X = In, Sn; Sb) exhibits a martensitic transformation in an off-stoichiometric composition range. ${ }^{111}$ In this category, MSMAs with low dimensionality have been steadily gaining interest and are indeed the most studied magnetocaloric materials at the micro- and nanoscale. Dunand and Mullner have reviewed this effort thoroughly, covering the major achievements in size-reduction the Ni-Mn-Ga system. ${ }^{112}$ In particular, they focus on the importance of substituting grain boundaries by surfaces (engineering $\mathrm{Ni}-\mathrm{Mn}-\mathrm{Ga}$ nanostructures with a typical size similar to grain size) enabling a free pathway for the twin boundary motion and consequently leading to colossal (2-10\%) magnetic field induced strains (MFIS). Several examples illustrate this principle, such as (i) clever introduction of pores smaller than the grain sizes in $\mathrm{Ni}-\mathrm{Mn}-\mathrm{Ga}$ polycrystalline sample by Chmielus and coworkers, enabling $2-3.5 \%$ MFIS that remain operational after more than 200,000 cycles $^{113}$; (ii) the $1 \%$ MFIS in a bamboo-like ensemble of fibers with $40-100 \mu \mathrm{m}$ diameter ${ }^{114}$; (iii) $0.15 \%$ MFIS in directional solidified plate-shaped samples. ${ }^{115}$ In addition, FM Heusler thin films have been highlighted in the literature as promising components in spintronic devices. ${ }^{110}$ Basically, phase stability, grain size, and surface effect are referred as the main differences between the bulk and thin films. Further interesting properties have been already found in thin films, such as a large MCE, giant magnetoresistance, and MFIS. ${ }^{10,112}$ As typically, the maximum values of these properties are usually obtained around martensitic transformation temperature, which for thin films can be affected by the substrate choice, ${ }^{116}$ size effect (grain size and thickness),${ }^{117}$ phase compatibility (between martensite-low temperature-and austenite-high temperature), and heat treatment procedures. ${ }^{118}$ It has been shown that the choice of the substrate is very important because it influences the thin film growth, possibly leading to a stress-induced martensite or a preferential austenite phase at the interface. ${ }^{111,119-122}$ Several substrates have been used so far 
in the deposition of FSMA thin films, such as silicon $(\mathrm{Si}),{ }^{118,123}$ aluminum oxide $\left(\mathrm{Al}_{2} \mathrm{O}_{3}\right),{ }^{124}$ magnesium oxide $(\mathrm{MgO}){ }^{111,120}$ strontium titanate (STO), ${ }^{125}$ and Yttriastabilized zirconia (YSZ). ${ }^{126}$ In the literature, the use of $\mathrm{MgO}(001)$ substrates is very common due to its low lattice mismatch with FSMA, enabling epitaxial growth (i.e., Ni-Mn based Heusler alloy thin films). As they grow with a preferential orientation, the reorientation mechanisms of epitaxial thin films will be affected. ${ }^{111}$ For example, for GaAS(001) and $\mathrm{Al}_{2} \mathrm{O}_{3}(110)$, Roytburd and coworkers found the austenite phase at room temperature, whereas for $\mathrm{MgO}(100)$, two phases (austenite or martensite) were observed. ${ }^{116}$

Other studies were focused on the influence of the thickness in the martensitic transformation in Heusler alloys and the existence of a critical size limit. Ranzieri et al. ${ }^{108}$ have investigated epitaxial $\mathrm{Ni}-\mathrm{Mn}-\mathrm{Ga}$ films on $\mathrm{MgO}\left(\begin{array}{lll}1 & 0 & 0\end{array}\right)$, of 10 and $100 \mathrm{~nm}$ thickness and have showed that martensitic transformations occur for thicknesses above $40 \mathrm{~nm}$. For nonepitaxial thin films, Vishnoi et al. ${ }^{123}$ studied the $\mathrm{Ni}-\mathrm{Mn}-\mathrm{Sn}$ on $\mathrm{Si}$ substrates in the thickness range of 120-2500 $\mathrm{nm}$ and found a suppression of the martensitic transformation below $410 \mathrm{~nm}$. More recently, Teichert et al. ${ }^{111}$ studied epitaxial $\mathrm{Ni}_{51.6} \mathrm{Mn}_{32.9} \mathrm{Sn}_{15.5}$ and $\mathrm{Ni}_{51.6} \mathrm{Mn}_{34.9} \mathrm{Sn}_{13.5}$ thin films on $\mathrm{MgO}\left(\begin{array}{lll}0 & 0 & 1\end{array}\right)$ in the thickness range of 10-200 $\mathrm{nm}$ and found that the thicker films (100 and $200 \mathrm{~nm}$ ) show bulklike transformation behavior, but when the film thickness is decreased, these transformations become less pronounced and the transformation temperatures also decrease, as shown in Fig. 6(a). In Fig. 6(b), the thickness dependence of the martensitic transformation and the $T_{\mathrm{C}}$ is evidenced. It is observed that for this composition, the austenite starting temperature $\left(A_{\mathrm{s}}\right)$ and finish martensite temperature $\left(M_{\mathrm{f}}\right)$ show a strong dependence on the film thickness.

Thermal treatments were also reported regarding the optimization of the magnetic and structural properties of the Heusler alloys. ${ }^{118,127}$ Kumar et al., ${ }^{118}$ for example, have produced $\mathrm{Ni}-\mathrm{Mn}-\mathrm{Ga}$ on $\mathrm{Si}(100)$ substrates using DC magnetron sputtering and studied postannealings in these films. The authors performed an annealing at $600{ }^{\circ} \mathrm{C}$ for $30 \mathrm{~min}$ and observed that: a FM ordering is recovered and the quasi-amorphous structure has changed to nanocrystalline structure. At low film thickness $(<300 \mathrm{~nm})$, the annealed films exhibited a mixture of cubic austenite (L21) and martensite phases, while at thicknesses greater than $1000 \mathrm{~nm}$, an ordered L12 phase was observed. The magnetic properties were found to strongly depend on the structure of the constituent phases present in the films. At higher thicknesses, annealed films presented weak FM ordering, as shown in Fig. $7{ }^{118}$ Note that recently the origin of the martensitic transition in the MSMA Ni-Mn-Ga was described by Schubert et al., ${ }^{128}$ like a complex charge-density wave tuned by magnetic ordering and strong electron-lattice coupling. Moreover
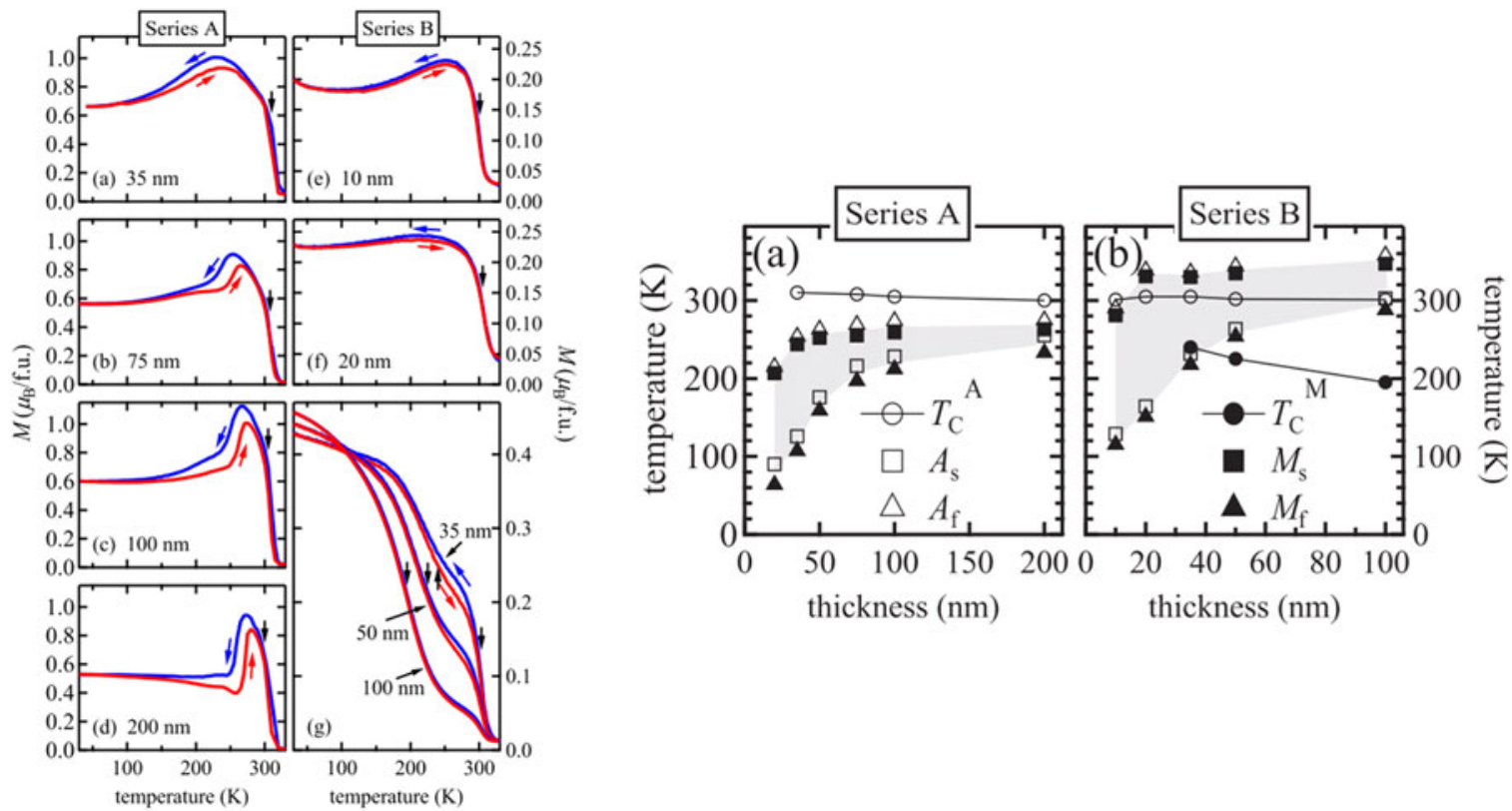

FIG. 6. To the left, $M(T)$ curves at low field for $\mathrm{Ni}_{51.6} \mathrm{Mn}_{32.9} \mathrm{Sn}_{15.5}$ (Series A) and $\mathrm{Ni}_{51.6} \mathrm{Mn}_{34.9} \mathrm{Sn}_{13.5}$ (Series B) onto MgO (0 01 ) thin film with different thicknesses and to the right, phase diagram of the two materials as a function of thickness. The blue arrow indicates field cooling and the red arrow field heating. Series A was measured with an applied field of $5 \mathrm{mT}$ and series B with $15 \mathrm{mT}$. Phase diagrams of the both series. Note that the light gray area shows the temperature range of the martensitic transformation. Reprinted from Ref. 112 with permission from Elsevier. 
Zhang et al. ${ }^{129}$ showed the advantage of tailoring the thin film composition so that the magnetostructural martensitic transformation and the FM ordering of the austenitic phase occurred simultaneously. The MCE of Ni-Mn-Ga films, while reduced in comparison with the bulk counterpart, still shows a large entropy change of $\Delta S^{\max }$ $\sim 8.5 \mathrm{~J} /(\mathrm{kg} \mathrm{K})$ for a $\Delta H=60 \mathrm{kOe}$ field change. ${ }^{130}$

With the aim of optimizing magnetic properties like MCE, several approaches have been undertaken, where doping is most common. Akkera et al. ${ }^{121}$ demonstrated that the aluminum $(\mathrm{Al})$ content in $\mathrm{N}_{49.8} \mathrm{Mn}_{32.97} \mathrm{Al}_{4.43} \mathrm{Sb}_{12.8}$ FSMA thin films leads to an increase in the martensitic transformation temperature. The authors established this correlation through temperature-dependent magnetization and resistance measurements and observed a higher $\Delta S^{\max }$ value of $23 \mathrm{~mJ} /\left(\mathrm{cm}^{3} \mathrm{~K}\right)$ at $300 \mathrm{~K}$ for a magnetic field change of $\Delta H=20 \mathrm{kOe}$ that is associated with a large magnetovolume coupling. ${ }^{121}$ Another work reported the study of different $\mathrm{Ni}-\mathrm{Co}-\mathrm{Mn}-\mathrm{Al}$ thin film compositions deposited onto $\mathrm{MgO}(001)$ substrates and concluded that the structure of the martensite phase is $14 \mathrm{M}$ and the metamagnetic martensite transformation occurs from the strongly FM austenite to the weakly magnetic martensite. ${ }^{131}$ The $\mathrm{Ni}_{41} \mathrm{Co}_{10.4} \mathrm{Mn}_{34.8} \mathrm{Al}_{13.8}$ films have shown an inverse GMCE with a peak value of $\Delta S^{\max }$ $\sim \mathrm{J} /(\mathrm{kg} \mathrm{K})$ for $\Delta H=50 \mathrm{kOe}^{131}$

Other important Heusler alloy is the Ni-Co-Mn-In alloy, whose magnetization increases on heating as a result of a structural transition, and consequently shows an inverse MCE. ${ }^{132}$ One advantage of growing these materials in thin film form is the potential of reducing the firstorder nature of their transition, due to the strong coupling to the substrate. Niemann et al. ${ }^{132}$ found that in epitaxial $\mathrm{Ni}-\mathrm{Co}-\mathrm{Mn}-\mathrm{In}$ on $\mathrm{MgO}\left(\begin{array}{lll}0 & 0 & 1\end{array}\right)$, the portion of the film immediately coupled to the substrate remained in the FM austenite phase, indicating that the coupling completely suppressed the martensitic transformation (Fig. 8). These films exhibit an inverse MCE with a maximum of $\Delta S^{\max }$ $\sim 8.8 \mathrm{~J} /(\mathrm{kg} \mathrm{K})$ at $353 \mathrm{~K}$, under a $\Delta H=90 \mathrm{kOe}$ applied field.

\section{H. $\mathrm{ABO}_{3}$ perovskites}

Magnetic oxides are another family presenting a strong coupling between spin and lattice degrees of freedom. The materials scientists' interest is due to their
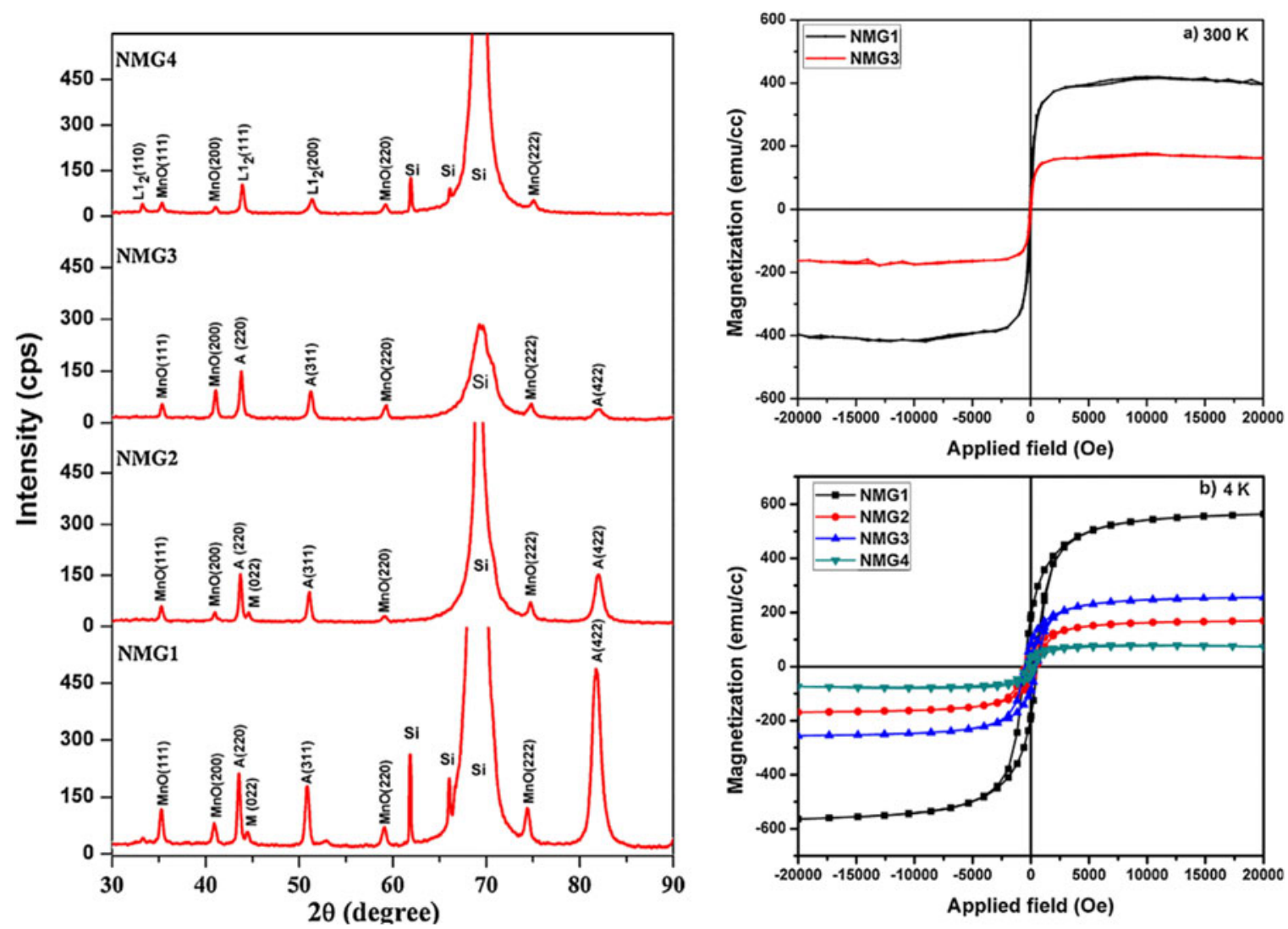

FIG. 7. Thickness-dependent magnetic properties and phase structure evolution in annealed Ni-Mn-Ga thin films. Thickness of NMG4 $>$ NMG1. Reprinted Ref. 119 with permission from Elsevier. 

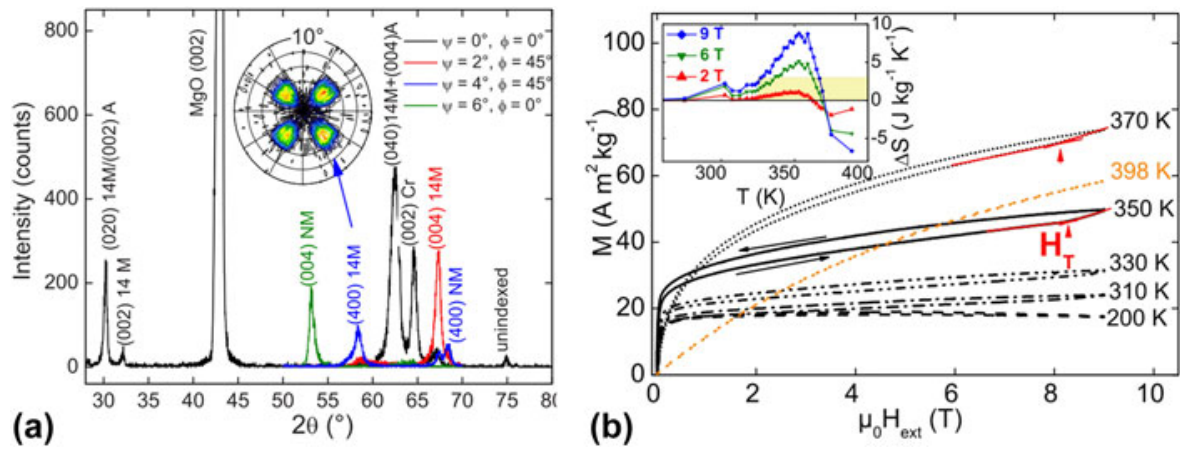

FIG. 8. (a) Four-circle XRD analyses of the martensitic state at room temperature by $\theta-2 \theta$-scans (Philips $\mathrm{X}^{\prime} \mathrm{Pert}, \mathrm{Cu} \mathrm{K}_{\alpha}, \lambda=0.15406 \mathrm{~nm}$ ). (b) Specific magnetization as a function of external field consecutively measured after undercooling to $50 \mathrm{~K}$. The inset shows the specific change in entropy calculated from all consecutive $M(H)$ measurements. Around the metamagnetic martensitic transition, a positive $\Delta S$ with a maximum of $8.8 \mathrm{~J} /(\mathrm{kg} \mathrm{K})$ at $353 \mathrm{~K}$ is observed. Reproduced from Ref. 132 with the permission of AIP Publishing.
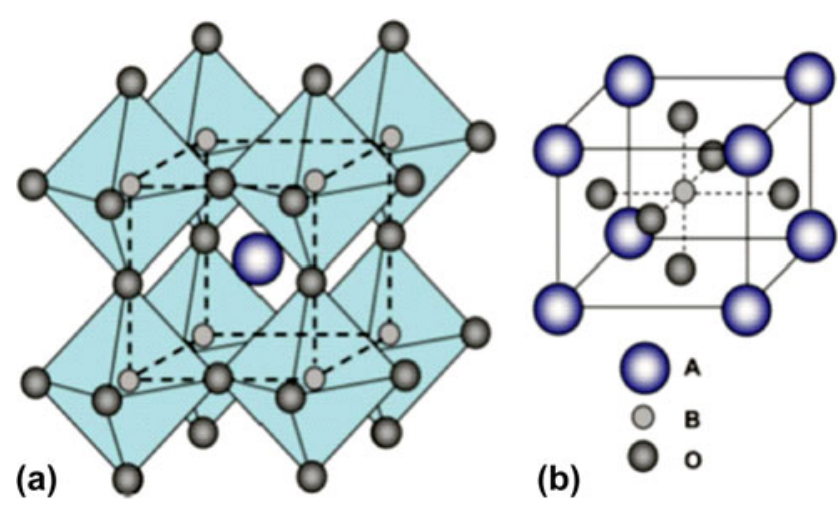

FIG. 9. Schematics of magnetic oxide heterostructures. Reproduced from Ref. 133 with permission of The Royal Society of Chemistry.

multifunctional nature, which made them widely studied and applied in today technological devices. In this section, few examples are given to show the facets of interesting physical properties of magnetic oxide thin films as well as their potential applications in areas such as electronics, spintronics, refrigeration, and strictive. Two of the most important families are $\mathrm{ABO}_{3}$ perovskites and $\mathrm{AB}_{2} \mathrm{O}_{4}$ spinels ( $\mathrm{A}$ and $\mathrm{B}$ can be $3 d$ or $4 f$ elements) as they are fertile in different forms of magnetism. Their rich and complex magnetism arises mainly due to the atomic structure that leads to new bonding geometries and their electronic states nature. Thus, the magnetic oxides' thin film nanostructures allow the exploration of the exchange interaction lengths and their tuning on an atomic length scale - fundamental for the emergence of new functionalities. In a cubic unit cell, type "A" atoms (violet spheres in Fig. 9) sit at cube corner positions ( 0,0 , 0 ), type "B" atoms (smaller and light gray spheres) sit at body-centered position $(1 / 2,1 / 2,1 / 2)$, and oxygen atoms (larger and darker gray spheres) sit at face-centered positions $(1 / 2,1 / 2,0)$. This arrangement can also be seen as a set of octahedra, where the " $\mathrm{B}$ " atoms lie in the center and are coordinated by six $\mathrm{O}$ atoms lying in the vertices. ${ }^{133}$

The interstitial A-sites are occupied by a cation ( $3 d$ or $4 f$ elements), whose oxidation state is dependent on the B-site transition metal. Also, the geometry plays an important role on the magnetism, namely, the $\mathrm{B}-\mathrm{O}-\mathrm{B}$ network, which forms a perfectly cubic $\mathrm{ABO}_{3}$ unit cell with $180^{\circ} \mathrm{B}-\mathrm{O}-\mathrm{B}$ bond angles. The $\mathrm{A}-\mathrm{O}$ and $\mathrm{B}-\mathrm{O}$ bond lengths are such that the Goldschmidt tolerance factor $t=$ $(\mathrm{rA}-\mathrm{rO}) /(\sqrt{2}(\mathrm{rB}+\mathrm{rO}))$ is $1.0,{ }^{134}$ where $\mathrm{rA}, \mathrm{rB}$, and $\mathrm{rO}$ are the radii of the A-cation, B-cation, and anion, respectively. For a large number of magnetic oxides, it is constrained to $0.71-1$, where the coordination between atoms and constraints of three-dimensional connectivity are accommodated by cooperative distortions such as tilts and rotations of the constituent octahedra. ${ }^{135}$ Thus, the pseudocubic structures, such as orthorhombic, tetragonal, or rhombohedra symmetries are observed for low distortions. Moreover, the change of the $\mathrm{B}-\mathrm{O}-\mathrm{B}$ bond angles leads to multiple competing magnetic interaction exchange (in direction and strength), which are mediated through these bonds. Another important parameter is the oxidation state of the B atom since it can also strongly influence the nature of the magnetic state. In special cases, B represents a mixed valence that leads to a doubleexchange interaction mechanism, which is mainly responsible for the appearance of the FM state. In the case of presenting only one magnetic state, the magnetic mechanism is called of super-exchange anti-FM or weak FM state-GKA rules proposed by Goodenough, ${ }^{136}$ Kanamori, ${ }^{137}$ and Anderson ${ }^{138}$ explained in detail this mechanism. Another important parameter that should be taken into account is the presence of structural distortions. These can lead to the appearance of Jahn-Teller effect, which in turn is responsible for the crystal field splitting ${ }^{135}$ and consequently, the mediation of the magnetic state.

Considering the scale reduction studies of this system of materials, one of the major achievements was carried 
out by $\mathrm{Jin}$ and co-authors in $1994,{ }^{139}$ in $\mathrm{La}_{1-x} \mathrm{Ca}_{x} \mathrm{MnO}_{3}$ (with $x \sim 0.67$ ), when they deposited a thin film by pulsed laser ablation exhibiting a negative isotropic magnetoresistance effect more than three orders of magnitude higher than the typical value observed in superlattice films. These epitaxial films exhibit magnetoresistance values as high as $127,000 \%$ near $77 \mathrm{~K}$ and approximately $1300 \%$ near room temperature. ${ }^{139}$ Furthermore, a thorough report by Lampen and coworkers highlighted the impact of size-reduction on samples with different typical sizes, namely, a $150 \mathrm{~nm}$ thick film deposited by PLD on top of the $\mathrm{MgO}$ substrate and two sets of sol-gel chemically prepared powders of 15 and $33 \mathrm{~nm}$ typical size. They remark the major impact of size-reduction: the broadening of the magnetic transition and the reduction of the $T_{\mathrm{C}}$, magnetic moment, and $\Delta S^{\max }$ associated with a weakening of the magnetic transition first-order nature. ${ }^{140}$ Wang et al. ${ }^{141}$ in 2002 were one of the first to study the MCE of a laser pulsed deposited $\mathrm{La}_{0.78} \mathrm{Ag}_{0.22} \mathrm{MnO}_{3}$ thin film, reporting $\Delta S^{\max } \sim$ $-2.22 \mathrm{~J} /(\mathrm{kg} \mathrm{K})$ at room temperature under $\Delta H=10 \mathrm{kOe}$ (Fig. 10).

In 2009, a thorough study of the MCE was performed in $\mathrm{La}_{0.75} \mathrm{Ba}_{0.1} \mathrm{M}_{0.15} \mathrm{MnO}_{3}(\mathrm{M}=\mathrm{Na}, \mathrm{Ag}$, and $\mathrm{K})$ thin films. The values achieved were $\Delta S^{\max } \sim 2.26,1.72$, and $2.44 \mathrm{~J} /(\mathrm{kg} \mathrm{K})$, respectively, under a magnetic field change of $\Delta H=20 \mathrm{kOe}^{142}$ In 2011, Prellier et al. ${ }^{143}$ grew a series of $\left(\mathrm{La}_{0.7} \mathrm{Sr}_{0.3} \mathrm{MnO}_{3} / \mathrm{SrRuO}_{3}\right)$ superlattices, where the effect of varying $\mathrm{SrRuO}_{3}$ layer thickness was examined. A room-temperature MCE was obtained owing to the finite size effect, which reduced the $T_{\mathrm{C}}$ of $\mathrm{La}_{0.7} \mathrm{Sr}_{0.3} \mathrm{MnO}_{3}$ layers. While the working temperature ranges were enlarged, the $-\Delta S^{\max }$ values remained similar to the values in polycrystalline $\mathrm{La}_{0.7} \mathrm{Sr}_{0.3} \mathrm{MnO}_{3}$. Consequently, the RCPs were significantly improved, the microscopic mechanism that is related to the effect at the

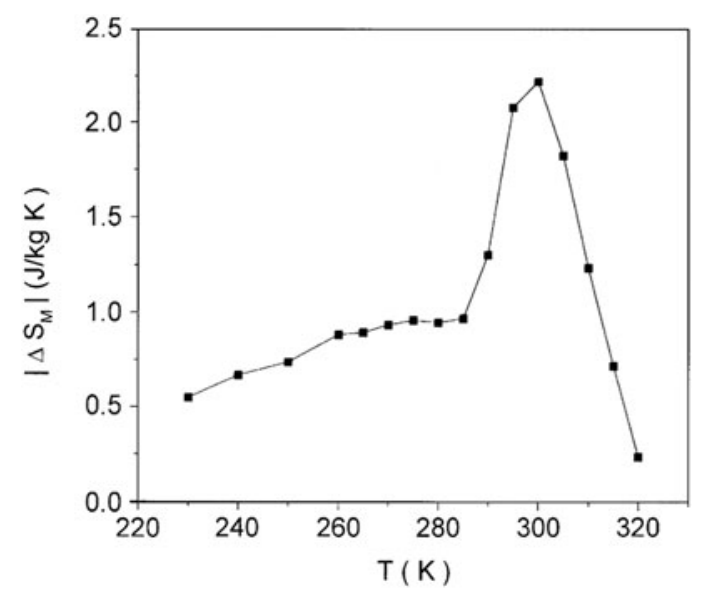

FIG. 10. Magnetic entropy change maximum obtained by Wang et al. under a magnetic field of $1 \mathrm{~T}$ as a function of the temperature. Reprinted from Ref. 141 with permission from Elsevier. interfaces at $\mathrm{La}_{0.7} \mathrm{Sr}_{0.3} \mathrm{MnO}_{3} / \mathrm{SrRuO}_{3}$, and higher nanostructural disorder. This pioneering study indicated that artificial oxide superlattices/multilayers might provide an alternative pathway in the search for efficient roomtemperature magnetic refrigerators for (nano) microscale systems. ${ }^{19,144}$

Other epitaxial thin films were presented in 2012 by Goktas et al. ${ }^{145}$ when they deposited manganite $\mathrm{La}_{0.67} \mathrm{Ag}_{0.33} \mathrm{MnO}_{3}$ (LAgMO) and $\mathrm{La}_{0.67} \mathrm{~K}_{0.33} \mathrm{MnO}_{3}$ (LKMO) films on the top of $\mathrm{LaAlO}_{3}\left(\begin{array}{lll}1 & 0 & 0\end{array}\right)$ and quartz substrates by using the sol-gel dip-coating technique. This work showed that the LAgMO and LKMO epitaxial films present different metal-insulator transition temperatures (T-MI) and PM-FM phase transition temperatures $\left(T_{\mathrm{C}}\right){ }^{145}$ In the same year, Belyea and co-authors ${ }^{144}$ investigated the MCE of two epitaxial multilayer manganite heterostructures following the strategy of Prellier work, ${ }^{143}$ i.e., a multilayer composed by a single layer of the $\mathrm{La}_{0.56} \mathrm{Sr}_{0.44} \mathrm{MnO}_{3}$ alloy with randomly distributed $\mathrm{La}$ and $\mathrm{Sr}$ cations, the other a digitally synthesized superlattice of $\mathrm{LaMnO}_{3}$ and $\mathrm{SrMnO}_{3}$ fabricated to be compositionally identical to the alloy. The magnetic entropy change and the RCP were larger for the alloy than for the superlattice, though both are suppressed relative to their bulk counterparts. These results indicate that the disorder of the A-site cation species in the perovskite structure may play a crucial role in defining the MCE in complex oxide materials. ${ }^{144}$

A major breakthrough was smartly engineered and successfully experimentally achieved by Moya et al. ${ }^{72}$ in 2013 when they presented a giant and reversible extrinsic MCE in the $\mathrm{La}_{0.7} \mathrm{Ca}_{0.3} \mathrm{MnO}_{3} / / \mathrm{BaTiO}_{3}$ thin film by using the strain-mediation between the substrate and the film near the substrate first-order structural phase transition temperature (Fig. 11). This extrinsic effect led to an impressive enhancement of $\Delta S_{\mathrm{m}}{ }^{\max }$ up to $9 \mathrm{~J} /(\mathrm{kg} \mathrm{K})$, ten times higher than the intrinsic effect observed in the $\mathrm{La}_{0.7} \mathrm{Ca}_{0.3} \mathrm{MnO}_{3}$ thin film $0.7 \mathrm{~J} /(\mathrm{kg} \mathrm{K})$ for the same $\Delta H=10 \mathrm{kOe}$.

In the same year, strain-dependent magnetocaloric properties of $\mathrm{La}_{0.67} \mathrm{Sr}_{0.33} \mathrm{MnO}_{3}$ thin films deposited on three different substrates: (l $\left.\begin{array}{lll}0 & 0 & 1\end{array}\right) \mathrm{LaAlO}_{3}$ (LAO), (l $\left.\begin{array}{lll}0 & 0 & 1\end{array}\right)$ $\mathrm{SrTiO}_{3}$ (STO), and $\left(\begin{array}{lll}0 & 0 & 1\end{array}\right) \mathrm{La}_{0.3} \mathrm{Sr}_{0.7} \mathrm{Al}_{0.65} \mathrm{Ta}_{0.35} \mathrm{O}_{9}$ (LSAT) corresponding to a less compressive (LAO), nearly lattice matched (LSAT) and largely tensile strained (LAO) have been investigated. Interestingly, the authors found that the sample with larger tensile strain (LSMO/ STO) was the one exhibiting higher RCP values, suggesting that strain can effectively tune the magnetocaloric properties in thin films and opening the possibility to use similar tool to tune the MCE in bulk samples too (Fig. 12). ${ }^{146}$

Similarly, Giri et al. ${ }^{147}$ deposited epitaxial $\mathrm{Sm}_{0.55} \mathrm{Sr}_{0.45} \mathrm{MnO}_{3}$ thin films onto the LAO (001), LSAT (001), and STO (001) single crystalline substrates by 

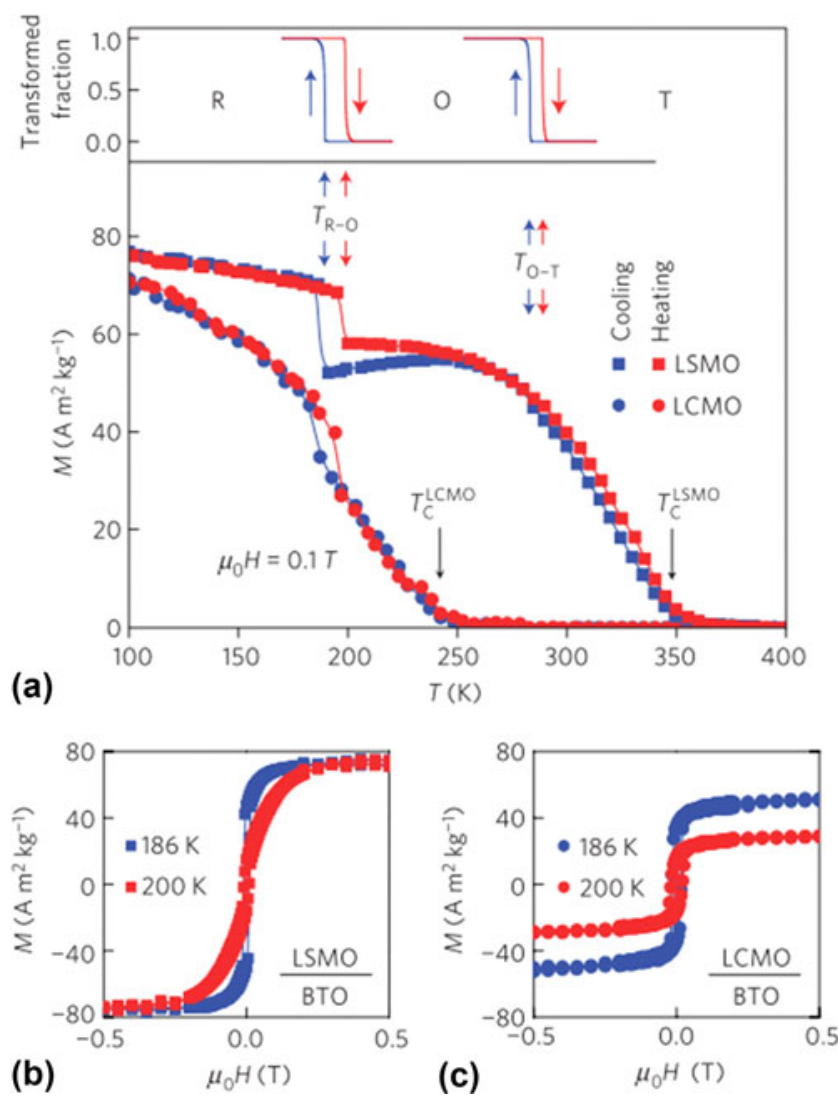

FIG. 11. $M(T)$ of LSMO/BTO and LCMO/BTO: (a) $M(T)$ measured on cooling (blue circles, LCMO; blue squares, LSMO) and heating (red circles, LCMO; red squares, LSMO), showing magnetic jumps near TR-O $\sim 200 \mathrm{~K}$ below film $T_{\mathrm{C}} \sim 350 \mathrm{~K}(\mathrm{LSMO})$ and $T_{\mathrm{C}} \sim 240 \mathrm{~K}$ (LCMO). (b) $M(H)$ for LSMO/BTO and (c) LCMO/BTO. Reprinted by permission from Ref. 72, copyright 2013.

pulsed laser deposition technique. They found that the film on the LAO substrate is under compressive strain and undergoes a transition at a Curie temperature of $165 \mathrm{~K}$. On the other end, the films on STO and LSAT substrates, which are under tensile strain, exhibited a Curie temperature at $120 \mathrm{~K}$ and $130 \mathrm{~K}$, respectively. Most interestingly, both normal (i.e., negative $\Delta S$ ) and inverse (i.e., positive $\Delta S$ ) MCEs have been observed around $T_{\mathrm{C}}$ and above their metal-insulator transition $\left(T_{\mathrm{p}}\right)$, respectively, for $\mathrm{Sm}_{0.55} \mathrm{Sr}_{0.45} \mathrm{MnO}_{3}$ thin films onto STO and LSAT substrates with a maximum value of MCE $\sim 10 \mathrm{~J} /(\mathrm{kg} \mathrm{K})$. The $\mathrm{Sm}_{0.55} \mathrm{Sr}_{0.45} \mathrm{MnO}_{3}$ on STO exhibits a large RCP of $142 \mathrm{~J} / \mathrm{kg}$ for a magnetic field change of $10 \mathrm{kOe}^{147}$

Other exotic spin-lattice related effects were observed while playing with the relative strain between the substrates and $\mathrm{La}_{1-x} \mathrm{Sr}_{x} \mathrm{MnO}_{3}$ films, such as anomalies in magnetostriction and thermal expansion, electric resistivity with a relevant dependence on film thickness, and the formation of magnetic stripe domains due to inhomogeneities and coexistence of different magnetic phases. ${ }^{94-97}$

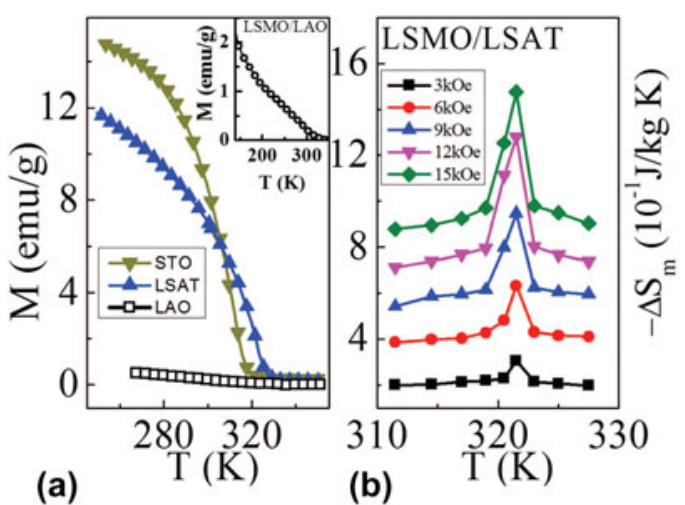

FIG. 12. (a) $M(T)$ for LSMO/STO, LSMO/LSAT, and LSMO/LAO under a field of $50 \mathrm{Oe}$, in the temperature range $250-350 \mathrm{~K}$ and (b) $\Delta S_{\mathrm{m}}$ for LSMO/LSAT in the temperature range $310 \mathrm{~K}<T<327 \mathrm{~K}$, under a field change of $1.5 \mathrm{~T}$. Reprinted from Ref. 146, licensed under a Creative Commons Attribution (CC BY) license.

Furthermore, the magnetovolume coupling of manganites and cobaltites thin film oxides have been thoroughly studied. Kundys and Szymczak ${ }^{148}$ explored the magnetostriction for both kind of thin films as a function of temperature and magnetic field. They showed that the giant magnetostriction in cobaltites arises from two phenomena: the orbital instability of $\mathrm{Co}^{3+}$ ions that under a magnetic field leads to a transition from a nondegenerated orbital low-spin state to a degenerated orbital intermediate-spin state and the increase of the FM cluster volume with the increase of magnetic field intensity. It seems that the last mechanism is common for all materials that present FM clusters embedded into nonFM matrix.

\section{TECHNOLOGICAL APPLICATIONS: MICRO- AND NANODEVICES}

As mentioned in Sec. I, the micro- and nanoscale open a new pathway for innovative technological applications which take advantage of the materials miniaturization. Rosensweig work ${ }^{149,150}$ was fundamental for the identification of several interesting applications for magnetocaloric fluids, composed of micro- and nanoparticles immersed in a liquid medium, long time before the thorough study of magnetocaloric micro- and nanostructures. Later, and following the path of Brown's room temperature magnetic refrigerator, ${ }^{151}$ Burnet published a patent of a room temperature magnetic refrigerator where instead of having a solid magnetocaloric material one would have a magnetocaloric ferrofluid, enabling a simpler machine design and potentially higher operational frequencies. ${ }^{152}$ The obvious miniaturization of refrigerators for cooling small volumes has also been explored. ${ }^{26-28,153,154}$ In 2001, Shirron's goal was to construct a $10 \mu \mathrm{W}$ refrigerator to continuously cool 
a $<10 \mathrm{~kg}$ mass load at sub-Kelvin temperature. ${ }^{153} \mathrm{Kim}$ and coworkers have constructed the first near room temperature magnetocaloric microcooler ${ }^{26}$ using $\mathrm{Gd}_{5} \mathrm{Si}_{2} \mathrm{Ge}_{2}$ bulk disc bonded to a $\mathrm{Si}$ chip, where microchannels were patterned, to attain a $7 \mathrm{~K}$ temperature change. On a different approach, Silva and coworkers proposed, by numerical simulations, an innovative solidstate microrefrigerator concept, whose main advantage is the absence of any liquid heat exchange medium and consequently enabling a much simpler design, as can be seen in Figs. 13(a) and 13(b). ${ }^{27}$ The numerical study resulted in a $2.75 \mathrm{~W} / \mathrm{cm}^{2}$ value for the coefficient of performance for an operating temperature around $296 \mathrm{~K}$ by simulating a Gd thin layer sandwiched by two thermal switches that are externally actuated by a magnetic field.

This concept was further explored by $\mathrm{Wu}$ and coworkers, who recently published their concept design [Fig. 13(c)] and a comprehensive simulation study of the geometrical parameters. Using gadolinium as the magnetocaloric material and an applied magnetic field of $15 \mathrm{kOe}$, a maximum no-load temperature span of $50.9 \mathrm{~K}$ is reached for a 72-lattice configuration.
Such refrigerators could have an important role on the cooling of the microprocessors, but also in other optical, electrical, or biological applications. This work has prompted the emergence of innovative thermal switch solutions such as those composed by a ferrofluid, ${ }^{19}$ thermoelectric materials, ${ }^{155}$ or magnetic materials whose thermal conductivity changes significantly upon the application of a magnetic field. For the latter case, materials presenting first-order transitions have great potential since drastic changes can be induced by a magnetic field on both the magnetic, structural, and electronic reservoirs of these materials, consequently leading to sharp variations on their thermal conductivity. Scarce thermal conductivity studies have been performed, namely, on the manganites sytem ${ }^{156,157}$ and on the $\mathrm{Fe}_{2} \mathrm{P}$ family of materials. ${ }^{157}$

The ferrofluid containing magnetic particles immersed in a liquid solution has been further developed for microfluidic pumps. ${ }^{29,158,159}$ The concept also introduced by Rosensweig is as follows: since magnetic materials lose their magnetization as the temperature approaches the material $T_{\mathrm{C}}$, then by exposing a column of magnetic

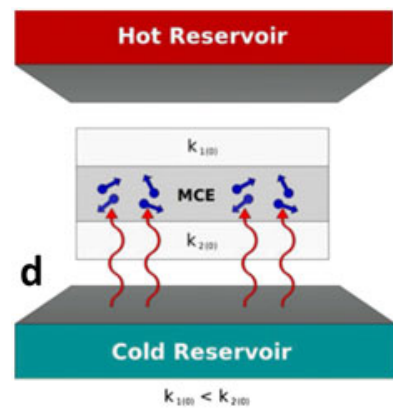

(a)

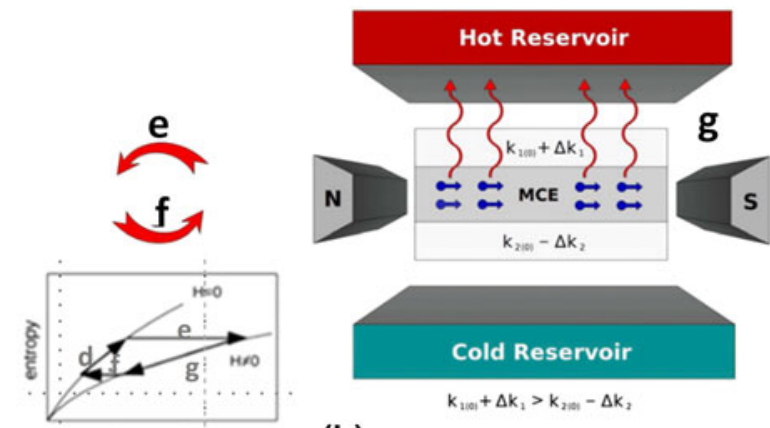

(b)

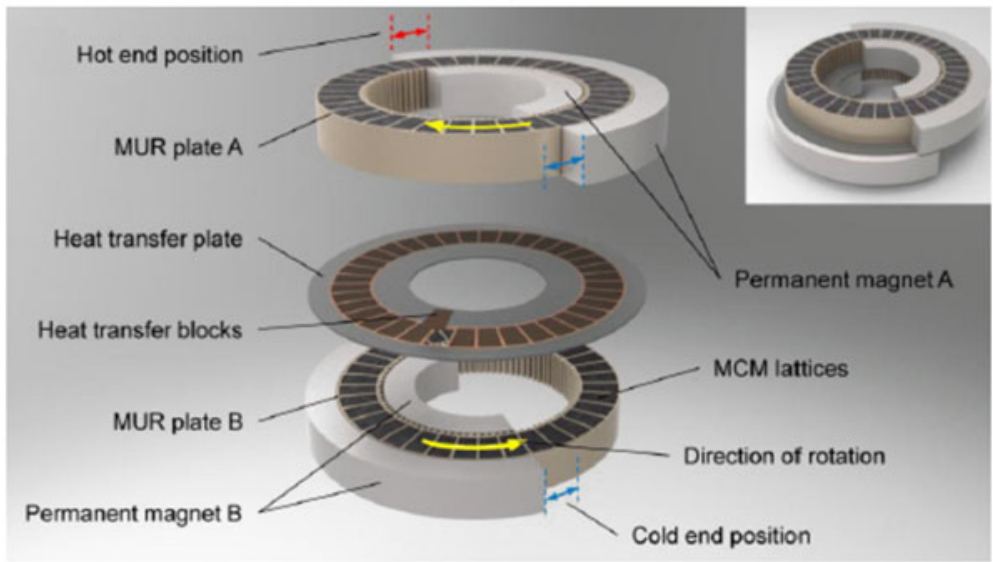

(c)

FIG. 13. ( $\mathrm{a}$ and $\mathrm{b}$ ) Proposed magnetic solid state device mechanism by Silva and coworkers. The two adiabatic processes occur during the application and removal of $H$ (e and f) while the two isofield processes take place during the heat flux between the cold reservoir and MCM (d) and between the MCM and the hot reservoir (g). The variation of the thermal conductivity with $H$ can establish a temperature gradient from the cold to the hot reservoir. The inset shows the entropy-temperature diagram of the Brayton cycle. Reproduced from Ref. 27 with the permission of Elsevier. (c) Rendering diagram of the novel solid-state magnetic refrigeration system proposed by Wu and coworkers. Reproduced from Ref. 28 with the permission of Elsevier. 


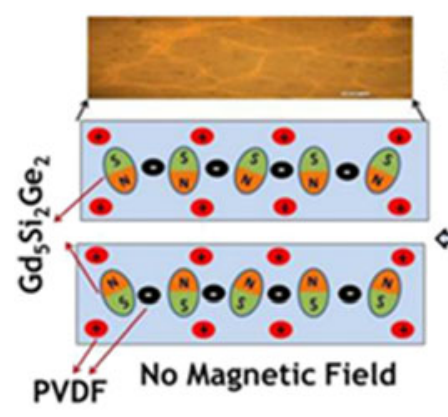

MFIVS: Magnetic Field Induced Volume Strain

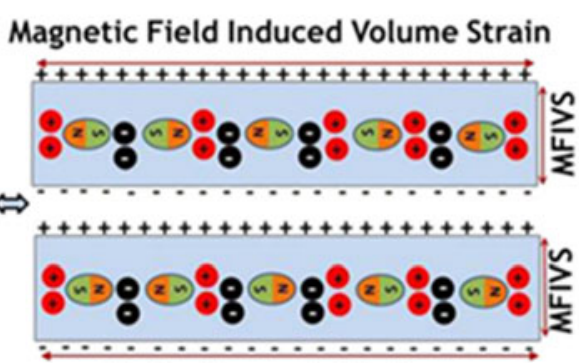

Under Magnetic Field

(a)

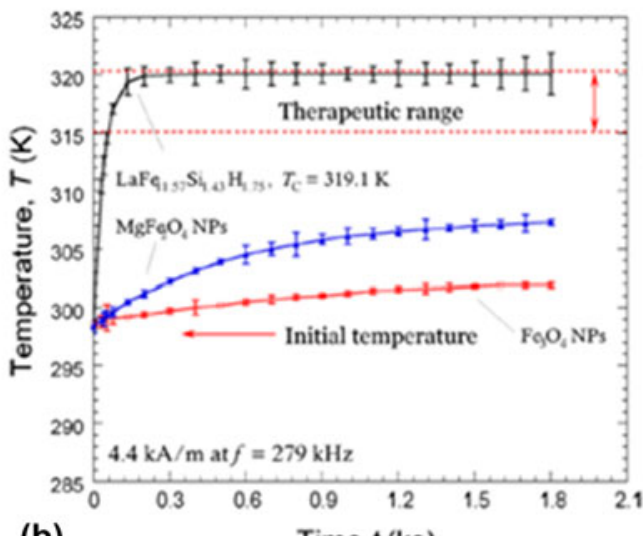

(b)

Time, $t(\mathbf{k s})$

FIG. 14. (a) Mechanism of energy conversion proposed by Ozaydin and Liang comprising a piezoelectric (PVDF) and a magnetostrictive material $\left(\mathrm{Gd}_{5} \mathrm{Si}_{2} \mathrm{Ge}_{2}\right)$. Reproduced from Ref. 32 with the permission of AIP. (b) Change in temperature as a function of induction heating time under $H=55$ Oe at $f$ $=279 \mathrm{kHz}$ for $\mathrm{LaFe}_{11.57} \mathrm{Si}_{1.43} \mathrm{H}_{1.75}, \mathrm{MgFe}_{2} \mathrm{O}_{4}$, and $\mathrm{Fe}_{3} \mathrm{O}_{4}$ suspensions. Reproduced from Ref. 162 with the permission of AIP.

fluid to a uniform magnetic field coincident with a temperature gradient will produce a pressure gradient in the magnetic fluid. As the fluid heats up, it loses its attraction to the magnetic field and is then displaced by the cooler fluid. Love and coworkers have thoroughly studied this system, including the ferrofluid synthesis and the pump design and performance. ${ }^{29}$ They highlight the advantage of not having movable mechanical parts in contrast with other microtechnologies such as piezoelectric, thermopneumatic, or shape memory actuation, where there is always a mechanical movement at high frequencies leading to short device duration. In particular, they stress that a magnetocaloric micropump would be particularly useful for lab-on-a-chip applications, where typically there is a requirement for thermal cycling for certain chemical processes, which could additionally control the microfluidic pump. The authors stress the importance of tailoring the magnetocaloric particles toward increased pyromagnetic coefficients (i.e., higher $\partial M / \partial T$ values). Since Love's report, the majority of the reports have been focused on the optimization of ferrite nanoparticles, whereas to our knowledge, there are no reports on the application of strong magnetovolume-coupled nanoparticles for this purpose.

The miniaturization of magnetocaloric materials and, in particular, of the strongly magnetovolume-coupled materials inspired applications in other areas such as energy harvesting or hyperthermia. Cleveland and coauthors have illustrated the energy harvesting/conversion concept by producing a composite material composed of a piezoelectric (PVDF) and a magnetocaloric material $\left(\mathrm{Gd}_{5} \mathrm{Si}_{2} \mathrm{Ge}_{2}\right) .{ }^{160}$ Even by using as small as 4 weight percent of the magnetocaloric material immersed in PVDF, the authors were able to collect $34.5 \mathrm{~W} / \mathrm{m}^{2}$ when the device was under a thermal cycle (from $-10{ }^{\circ} \mathrm{C}$ to $\left.+10{ }^{\circ} \mathrm{C}\right)$. Shortly after, Ozaydin and coworkers found promising results also for a similar composite for the conversion magnetic-to-electrical energy, ${ }^{32}$ as shown schematically in Fig. 14(a). Barkley and co-authors engineered another energy harvesting composite by mixing micron-sized particles of $\mathrm{Gd}_{5} \mathrm{Si}_{2} \mathrm{Ge}_{2}$ and aluminum toward tribological applications in automobile industry. ${ }^{161}$

The magnetocaloric materials miniaturization will certainly impact biotechnologies as well. Barati, Sandeman, and coworkers have recently explored the induction heating performance of the $\mathrm{La}-\mathrm{Fe}-\mathrm{Si}$ magnetocaloric material. ${ }^{162}$ As can be seen in Fig. 14(b), a suspension with $\mathrm{LaFe}_{11.57} \mathrm{Si}_{1.43} \mathrm{H}_{1.75}$ particles exhibits a much faster heating than $\mathrm{Fe}$-based suspensions, such as $\mathrm{MgFe}_{2} \mathrm{O}_{4}$ and $\mathrm{Fe}_{3} \mathrm{O}_{4}$, under the same alternating magnetic field intensity $(\sim 55 \mathrm{Oe})$ and frequency $(279 \mathrm{kHz})$. In addition, there is a temperature stabilization for the $\mathrm{La}-\mathrm{Fe}-\mathrm{Si}$ suspension at $T \sim 319 \mathrm{~K}$, lying within the therapeutic temperature window for hyperthermia treatments. The authors attribute the remarkable fast heating effect to the large magnetic hysteresis present in these materials and the temperature stabilization to their sharp magnetic transition at $T=T_{\mathrm{C}}=319 \mathrm{~K}$. Moreover, they envisage a promising route for $\mathrm{La}-\mathrm{Fe}-\mathrm{Si}$ particles in hyperthermia treatments due to their extraordinary induction heating performance and the ability to tune their Curie temperature by chemical stoichiometry.

\section{FINAL REMARKS AND OUTLOOK}

During the present review, a thorough review is presented concerning size-reduction (micro- and nanoscale) studies in the most promising magnetocaloric material families known to date. As clearly exemplified, this field is in the early stage and demands a thorough and methodic work. We emphasize several practical 
challenges arising at these scales, such as oxidation (few nm thick oxidation layer have a great impact on the final properties of a several $\mathrm{nm}$ thick magnetocaloric thin film), the optimization of the crystallinity; narrowing nanoparticles size-distribution; enhancing substrate-film interfaces; the disorder reduction, the tuning of $T_{\mathrm{C}}$, among others.

Despite being in its first steps, the micro- and nanoscale studies appear to have already achieved encouraging results aimed to (i) tackle the challenges of macroscopic magnetic refrigeration, (ii) help respond to the fundamental questions arising with size-reduction, and (iii) open new possibilities for micro- and nanodevices applications. Therefore, the recent years have shown that there is a fertile ground for micro- and nanoscale studies and this effort is expected to continue growing.

There are common features observed in the majority of these studies that seems to be generalized to all the nanosystems. Concerning the magnetic properties, there is a typical broadening of the magnetic transition and consequently a broadening of the $\Delta S(T)$ curve (enhancement of FWHM), the reduction of $\Delta S^{\max }$ and of the saturation magnetization. These effects are consequences of the higher impact that the disorder has in these smaller systems, namely: strain fields, atomic disorder, uncompensated surface spins, chemical inhomogeneities, grain size distribution, etc.

Another common factor to the majority of the studies is the enhanced role that stress plays in these systems, in particular, at nanoparticle surface or at the substrate-film interface, mimicking the effects of applying hydrostatic pressure in their bulk counterparts. This effect is more relevant in systems with strong magnetovolume coupling: for the $\mathrm{Gd}_{5}(\mathrm{Si}, \mathrm{Ge})_{4}$ nanoparticles, where the intrinsic surface stress leads to a $T_{\mathrm{C}}$ increase, and the manganite$\mathrm{BaTiO}_{3}$ film-substrate, where the structural transition in the substrate leads to an extrinsic large MCE-are good examples of the new underlying mechanisms arising at these scales.

The manganite system and the FSMAs are the two material families that have been explored in greater detail from the nano- (nanoparticles, nanowires) to the microscale (microstructure engineering). By contrast and surprisingly, few reports on the size-reduction of the La-Fe$\mathrm{Si}$ and the $\mathrm{Mn}-\mathrm{Fe}-\mathrm{P}-\mathrm{Si}$ materials have been published until now and therefore it is expected a significant increase in the following years. Whereas, the $\mathrm{Fe}-\mathrm{Rh}$ system has presented itself as one of the most proficuous playgrounds for material scientists at reduced scales, with surprising results such as the suppression of its irreversibility in the thin film form.

There is a promising future ahead for the micro- and nanostructuring of strong magnetovolume coupled materials that can be unfolded in three main branches: help tackling the challenges observed nowadays on macroscale magnetic refrigeration, investigate the role of the different underlying physical mechanisms emerging at these reduced dimensions, and drive the development of new application devices.

Finally, from the technological point of view, the uniqueness ability of producing nanomaterials will open a wide range of new applications such as microrefrigeration, microfluidic pumps, thermal switches, multiferroic devices, energy harvesting, drug delivery, and hyperthermia. These new fields expand the possible applications for magnetocaloric/or strongly magnetovolume coupled materials at reduced dimensions and consequently will attract an increasing number of material scientists and engineers to develop further the seminal ideas planted so far and potentially engineer new applications in the existing and in other fields. Finally, it is expected that new functionalities and devices will emerge when the fabrication of the materials and their control at the microand nanoscale is in a more mature state.

\section{ACKNOWLEDGMENTS}

The authors acknowledge Fundação para a Ciência e a Tecnologia for financial support through the projects: CERN/FIS-NUC/0004/2015, EXPL/EMS-ENE/2315/ 2013, and FEDER/POCTIn0155/94. J.H. Belo thanks FCT for the Grant SFRH/BD/88440/2012 and CICECOAveiro Institute of Materials, POCI-01-0145-FEDER007679 (FCT Ref. UID /CTM /50011/2013), financed by national funds through the FCT/MEC and when appropriate co-financed by FEDER under the PT2020 Partnership Agreement. A.M. Pereira acknowledges the project NORTE-070124-FEDER-000070 for financial support.

\section{REFERENCES}

1. C. Zimm, A. Jastrab, A. Sternberg, V. Pecharsky, K. Gschneidner, M. Osborne, and I. Anderson: Description and performance of a near-room temperature magnetic refrigerator. In Advances in Cryogenic Engineering, Vol. 43, P. Kittel, ed.; Pts A and B Advances in Cryogenic Engineering, Vol. 43 (Springer, Boston, MA, 1998); pp. 1759-1766.

2. V.K. Pecharsky and K.A. Gschneidner: Giant magnetocaloric effect in $\mathrm{Gd}_{5}\left(\mathrm{Si}_{2} \mathrm{Ge}_{2}\right)$. Phys. Rev. Lett. 78, 3-6 (1997).

3. A. Kitanovski, J. Tušek, U. Tomc, U. Plaznik, M. Ožbolt, and A. Poredoš: Magnetocaloric Energy Conversion (Springer International Publishing, Cham, 2015).

4. V. Franco, J.S. Blázquez, B. Ingale, and A. Conde: The magnetocaloric effect and magnetic refrigeration near room temperature: Materials and models. Annu. Rev. Mater. Res. 42, 305-342 (2012).

5. J. Liu, T. Gottschall, K.P. Skokov, J.D. Moore, and O. Gutfleisch: Giant magnetocaloric effect driven by structural transitions. Nat. Mater. 11, 620-626 (2012).

6. V.K. Pecharsky, K.A. Gschneidner, Jr., Y. Mudryk, and D. Paudyal: Making the most of the magnetic and lattice entropy changes. J. Magn. Magn. Mater. 321, 3541-3547 (2009). 
7. L. Morellon, P.A. Algarabel, M.R. Ibarra, J. Blasco, B. GarcíaLanda, Z. Arnold, and F. Albertini: Magnetic-field-induced structural phase transition in $\mathrm{Gd}_{5} \mathrm{Si}_{1.8} \mathrm{Ge}_{2.2}$. Phys. Rev. B 58, 721-724 (1998).

8. M.P. Annaorazov, S.A. Nikitin, A.L. Tyurin, K.A. Asatryan, and A.K. Dovletov: Anomalously high entropy change in FeRh alloy. J. Appl. Phys. 79, 1689-1695 (1996).

9. S. Fujieda, A. Fujita, and K. Fukamichi: Large magnetocaloric effect in $\mathrm{La}\left(\mathrm{Fe}_{x} \mathrm{Si}_{1-x}\right)_{13}$ itinerant-electron metamagnetic compounds. Appl. Phys. Lett. 81, 1276-1278 (2002).

10. F. Hu, B. Shen, and J. Sun: Magnetic entropy change in $\mathrm{Ni}_{51.5} \mathrm{Mn}_{22.7} \mathrm{Ga}_{25.8}$ alloy. Appl. Phys. Lett. 76, 3460-3462 (2000).

11. H. Wada and Y. Tanabe: Giant magnetocaloric effect of $\mathrm{MnAs}_{1-x} \mathrm{Sb}_{x}$. Appl. Phys. Lett. 79, 3302 (2001).

12. J. Mira, J. Rivas, L.E. Hueso, F. Rivadulla, and M.A. Lopez Quintela: Drop of magnetocaloric effect related to the change from first- to second-order magnetic phase transition in $\mathrm{La}_{2 / 3}$ $\left(\mathrm{Ca}_{1-x} \mathrm{Sr}_{x}\right)_{1 / 3} \mathrm{MnO}_{3}$. J. Appl. Phys. 91, 8903-8905 (2002).

13. F. Guillou, A.K. Pathak, D. Paudyal, Y. Mudryk, F. Wilhelm, A. Rogalev, and V.K. Pecharsky: Non-hysteretic first-order phase transition with large latent heat and giant low-field magnetocaloric effect. Nat. Commun. 9, 2925 (2018).

14. Y. Liu, L.C. Phillips, R. Mattana, M. Bibes, A. Barthélémy, and B. Dkhil: Large reversible caloric effect in FeRh thin films via a dual-stimulus multicaloric cycle. Nat. Commun. 7, 11614 (2016).

15. V. Provenzano, A.J. Shapiro, and R.D. Shull: Reduction of hysteresis losses in the magnetic refrigerant $\mathrm{Gd}_{5} \mathrm{Ge}_{2} \mathrm{Si}_{2}$ by the addition of iron. Nature 429, 853-857 (2004).

16. J.H. Belo, A.M. Pereira, J.P. Araujo, C. de la Cruz, A.M. dos Santos, J.N. Goncalves, V.S. Amaral, L. Morellon, P.A. Algarabel, and M.R. Ibarra: Tailoring the magnetism of $\mathrm{Tb}_{5} \mathrm{Si}_{2} \mathrm{Ge}_{2}$ compounds by La substitution. Phys. Rev. B 86, 14403-14412 (2012).

17. A. Fujita, S. Koiwai, S. Fujieda, K. Fukamichi, T. Kobayashi, H. Tsuji, S. Kaji, and A.T. Saito: Magnetocaloric effect in spherical $\mathrm{La}\left(\mathrm{Fe}_{x} \mathrm{Si}_{1-x}\right)_{13}$ and their hydrides for active magnetic regenerator-type refrigerator. J. Appl. Phys. 105, 07A93607A936-3 (2009).

18. T. Morikawa and H. Wada: Effect of deviation from stoichiometry on magnetic and magnetocaloric properties in $\mathrm{MnAs}_{1-x} \mathrm{Sb}_{x}$. J. Magn. Magn. Mater. 272, E583-E584 (2004).

19. J.B. Puga, B.D. Bordalo, D.J. Silva, M.M. Dias, J.H. Belo, J.P. Araújo, J.C.R.E. Oliveira, A.M. Pereira, and J. Ventura: Novel thermal switch based on magnetic nanofluids with remote activation. Nano Energy 31, 278-285 (2017).

20. G. Wehmeyer, T. Yabuki, C. Monachon, J. Wu, and C. Dames: Thermal diodes, regulators, and switches: Physical mechanisms and potential applications. Appl. Phys. Rev. 4, 041304 (2017).

21. H. Zhang, B.G. Shen, Z.Y. Xu, X.Q. Zheng, J. Shen, F.X. Hu, J.R. Sun, and Y. Long: Reduction of hysteresis loss and large magnetocaloric effect in the $\mathrm{C}$ - and $\mathrm{H}$-doped $\mathrm{La}(\mathrm{Fe}, \mathrm{Si})_{13}$ compounds around room temperature. J. Appl. Phys. 111, 1-4 (2012).

22. I.A. Radulov, D.Y. Karpenkov, K.P. Skokov, A.Y. Karpenkov, T. Braun, V. Brabänder, T. Gottschall, M. Pabst, B. Stoll, and O. Gutfleisch: Production and properties of metal-bonded $\mathrm{La}(\mathrm{Fe}$, $\mathrm{Mn}, \mathrm{Si})_{13} \mathrm{H}_{x}$ composite material. Acta Mater 127, 389-399 (2017).

23. I.A. Radulov, K.P. Skokov, D.Y. Karpenkov, T. Braun, O. Gutfleisch, and T.U. Darmstadt: Polymer-bonded La(Fe,Mn, $\mathrm{Si}_{13} \mathrm{H}_{x}$ plates for heat exchangers. IEEE Trans. Magn. 51, 11-14 (2015).

24. I. Radulov, D.Y. Karpenkov, M. Specht, T. Braun, A.Y. Karpenkov, K.P. Skokov, and O. Gutfleisch: Heat exchangers from metal bonded $\mathrm{La}(\mathrm{Fe}, \mathrm{Mn}, \mathrm{Si})_{13} \mathrm{H}_{x}$. Powder IEEE Trans. Magn. 53, 1 (2017).

25. J.D. Moore, G.K. Perkins, Y. Bugoslavsky, M.K. Chattopadhyay, S.B. Roy, P. Chaddah, V.K. Pecharsky, K.A. Gschneidner, Jr., and L.F. Cohen: Reducing the operational magnetic field in the prototype magnetocaloric system $\mathrm{Gd}_{5} \mathrm{Ge}_{4}$ by approaching the single cluster size limit. Appl. Phys. Lett. 88, 72501 (2006)

26. S. Kim, S. Ghirlanda, C. Adams, B. Bethala, S.N. Sambandam, and S. Bhansali: Design, fabrication and thermal characterization of a magnetocaloric microcooler. Int. J. Energy Res. 31, 717-727 (2007).

27. D.J. Silva, B.D. Bordalo, A.M. Pereira, J. Ventura, and J.P. Araújo: Solid state magnetic refrigerator. Appl. Energy 93, 570-574 (2012).

28. J. Wu, B. Lu, C. Liu, and J. He: A novel cascade micro-unit regeneration cycle for solid state magnetic refrigeration. Appl. Therm. Eng. 137, 836-847 (2018).

29. L.J. Love, J.F. Jansen, T.E. McKnight, Y. Roh, and T.J. Phelps: A magnetocaloric pump for microfluidic applications. IEEE Trans. Nanobioscience 3, 101-110 (2004).

30. S. Pal, A. Datta, S. Sen, A. Mukhopdhyay, K. Bandopadhyay, and R. Ganguly: Characterization of a ferrofluid-based thermomagnetic pump for microfluidic applications. J. Magn. Magn. Mater. 323, 2701-2709 (2011).

31. A. Kitanovski and P.W. Egolf: Innovative ideas for future research on magnetocaloric technologies. Int. J. Refrig. 33 449-464 (2010).

32. M.F. Ozaydin and H. Liang: Multi-energy conversion of $\mathrm{Gd}_{5}$ $\left(\mathrm{Si}_{2} \mathrm{Ge}_{2}\right)$-poly(vinylidene fluoride), a hybrid material. Appl. Phys. Lett. 105, 062903 (2014).

33. A.M. Tishin, Y.I. Spichkin, V.I. Zverev, and P.W. Egolf: A review and new perspectives for the magnetocaloric effect: New materials and local heating and cooling inside the human body. Int. J. Refrig. 68, 177-186 (2016).

34. C.W. Miller, D.D. Belyea, and B.J. Kirby: Magnetocaloric effect in nanoscale thin films and heterostructures. J. Vac. Sci. Technol., A 32, 040802 (2014)

35. G.J. Miller: Complex rare-earth tetrelides, $\mathrm{RE}_{5}\left(\mathrm{Si}_{x} \mathrm{Ge}_{1-x}\right)_{4}$ : New materials for magnetic refrigeration and a superb playground for solid state chemistry. Chem. Soc. Rev. 35, 799-813 (2006).

36. J. Lyubina: Magnetocaloric materials for energy efficient cooling. J. Phys. D: Appl. Phys. 50, 053002 (2017).

37. J.S. Amaral and V.S. Amaral: On estimating the magnetocaloric effect from magnetization measurements. J. Magn. Magn. Mater. 322, 1552-1557 (2010).

38. A.M. Tishin and Y.I. Spichkin: The Magnetocaloric Effect and its Applications (Institute of Physics Publishing, Bristol, Philadelphia, 2003).

39. V.K. Pecharsky and J.K. Gschneidner: Some common misconceptions concerning magnetic refrigerant materials. J. Appl. Phys. 90, 4614-4622 (2001).

40. H.F. Kirby, D.D. Belyea, J.T. Willman, and C.W. Miller: Effects of preparation conditions on the magnetocaloric properties of $\mathrm{Gd}$ thin films. J. Vac. Sci. Technol., A 31, 031506 (2013).

41. G. Scheunert, W.R. Hendren, C. Ward, and R.M. Bowman Magnetization of $2.6 \mathrm{~T}$ in gadolinium thin films. Appl. Phys. Lett. 101, 142407 (2012).

42. P.J. von Ranke, N.A. de Oliveira, V.S.R. de Sousa, D.C. Garcia, I.G. de Oliveira, A.M.G. Carvalho, and S. Gama: The influence of the spin reorientation process on the magnetocaloric effect: Application to $\mathrm{PrAl}_{2}$. J. Magn. Magn. Mater. 313, 176-181 (2007).

43. G. Scheunert, C. Ward, W.R. Hendren, A.A. Lapicki, R. Hardeman, M. Mooney, M. Gubbins, and R.M. Bowman: Influence of strain and polycrystalline ordering on magnetic 
properties of high moment rare earth metals and alloys. J. Phys. D: Appl. Phys. 47, 415005 (2014).

44. Y. Yamada, M. Okada, P. Jin, M. Tazawa, and K. Yoshimura: The Curie temperature dependence on preparation conditions for Gd thin films. Thin Solid Films 459, 191-194 (2004).

45. S.P. Mathew, S.N. Kaul, A.K. Nigam, A-C. Probst, and R. Birringer: Magnetic irreversibility, spin-wave excitations and magnetocaloric effect in nanocrystalline Gadolinium International Conference on Magnetism (ICM 2009). J. Phys. Conf. 200, 072047-1-072047-4 (2010).

46. B.I. Aruna, B.R. Mehta, L.K. Malhotra, and S.M. Shivaprasad: Stability and hydrogenation of bare gadolinium nanoparticles. Adv. Funct. Mater. 15, 131-137 (2005).

47. Y.G. Morozov, A.N. Kostygov, V.I. Petinov, and P.E. Chizhov: Disappearance of magnetic ordering in fine gadolinium particles. Phys. Status Solidi 32, K119-K121 (1975).

48. Y.N. Ertas, N.N. Jarenwattananon, and L.S. Bouchard: Oxidefree gadolinium nanocrystals with large magnetic moments. Chem. Mater. 27, 5371-5376 (2015).

49. H. Zeng, J. Zhang, C. Kuang, and M. Yue: Magnetic entropy change in bulk nanocrystalline Gd metals. Appl. Nanosci. 1, 51-57 (2011).

50. J.S. Amaral and V.S. Amaral: Disorder effects in giant magnetocaloric materials. Phys. Status Solidi 211, 971-974 (2014).

51. D.H.A.L. Anselmo, V.D. Mello, and M.S. Vasconcelos: Simulation of the magnetocaloric effect in Tb nanofilms. AIP Conf. Proc. 79, 79-81 (2014).

52. F.C.M. Filho, V.D. Mello, A.L. Dantas, F.H.S. Sales, and A.S. Carriço: Giant magnetocaloric effect of thin Ho films. $J$. Appl. Phys. 109, 07A914 (2011).

53. V.D. Mello, A.L. Dantas, and A.S. Carriço: Magnetocaloric effect of thin Dy films. Solid State Commun. 140, 447-451 (2006).

54. Y.Z. Shao, J.K.L. Lai, and C.H. Shek: Preparation of nanocomposite working substances for room-temperature magnetic refrigeration. J. Magn. Magn. Mater. 163, 103-108 (1996).

55. V. Franco, K.R. Pirota, V.M. Prida, A.M.J.C. Neto, A. Conde, M. Knobel, B. Hernando, and M. Vazquez: Tailoring of magnetocaloric response in nanostructured materials: Role of anisotropy. Phys. Rev. B 77, 104434-1-104434-7 (2008).

56. K.A. Gschneidner, Jr., V.K. Pecharsky, and A.O. Tsokol: Recent developments in magnetocaloric materials. Reports Prog. Phys. 68, 1479-1539 (2005)

57. L. Morellon, J. Stankiewicz, B. García-Landa, P.A. Algarabel, and M.R. Ibarra: Giant magnetoresistance near the magnetostructural transition in $\mathrm{Gd}_{5}\left(\mathrm{Si}_{1.8} \mathrm{Ge}_{2.2}\right)$. Appl. Phys. Lett. 73, 3462 (1998).

58. R.L. Hadimani, P.A. Bartlett, Y. Melikhov, J.E. Snyder, and D.C. Jiles: Field and temperature induced colossal strain in $\mathrm{Gd}_{5}$ $\left(\mathrm{Si}_{x} \mathrm{Ge}_{1-x}\right)_{4}$. J. Magn. Magn. Mater. 323, 532-534 (2011).

59. P.V. Trevizoli, C.S. Alves, M.A.B. Mendes, A.M.G. Carvalho, and S. Gama: Powder metallurgy influences on the magnetic properties of $\mathrm{Gd}_{5.09} \mathrm{Ge}_{2.03} \mathrm{Si}_{188}$ alloy. J. Magn. Magn. Mater. 320, 1582-1585 (2008).

60. J.S. Blázquez, J.J. Ipus, L.M. Moreno-Ramírez, J.M. ÁlvarezGómez, D. Sánchez-Jiménez, S. Lozano-Pérez, V. Franco, and A. Conde: Ball milling as a way to produce magnetic and magnetocaloric materials: A review. J. Mater. Sci. 52, 11834 11850 (2017).

61. A.L. Pires, J.H. Belo, J. Turcaud, G.N.P. Oliveira, J.P. Araújo, A. Berenov, L.F. Cohen, A.M.L. Lopes, and A.M. Pereira: Influence of short time milling in $\mathrm{R} 5(\mathrm{Si}, \mathrm{Ge})_{4}, \mathrm{R}=\mathrm{Gd}$ and $\mathrm{Tb}$, magnetocaloric materials. Mater. Des. 85, 32-38 (2015).

62. G. Giovanna, V. Svitlyk, M. Jafelicci, and Y. Mozharivskyj: Bulk and high-energy ball-milled $\mathrm{Gd}_{5} \mathrm{Si}_{2} \mathrm{Ge}_{2}$ : Comparative study of magnetic and magnetocaloric properties. Solid State Sci. $\mathbf{1 3}$ 209-215 (2011).
63. D.M. Rajkumar, M.M. Raja, R. Gopalan, and V. Chandrasekaran: Magnetocaloric effect in high-energy ballmilled $\mathrm{Gd}_{5} \mathrm{Si}_{2} \mathrm{Ge}_{2}$ and $\mathrm{Gd}_{5} \mathrm{Si}_{2} \mathrm{Ge}_{2} / \mathrm{Fe}$ nanopowders. J. Magn. Magn. Mater. 320, 1479-1484 (2008).

64. S.N. Sambandam, B. Bethala, D.K. Sood, and S. Bhansali: Evaluation of silicon nitride as a diffusion barrier for $\mathrm{Gd}-\mathrm{Si}-\mathrm{Ge}$ films on silicon. Surf. Coatings Technol. 200, 1335-1340 (2005)

65. R.L. Hadimani, I.C. Nlebedim, Y. Melikhov, and D.C. Jiles: Growth and characterisation of $\mathrm{Gd}_{5}\left(\mathrm{Si}_{x} \mathrm{Ge}_{1-x}\right)_{4}$ thin film. J. Appl. Phys. 113, 17A935 (2013).

66. R.L. Hadimani, J.H.B. Silva, A.M. Pereira, D.L. Schlagel, T.A. Lograsso, Y. Ren, D.C. Jiles, and J.P. Araújo: Gd5(Si,Ge $)_{4}$ thin film displaying large magnetocaloric and strain effects due to magnetostructural transition. Appl. Phys. Lett. 5, 32402 (2015).

67. A.L. Pires, J.H. Belo, I.T. Gomes, A.M.L. Lopes, J.P. Araújo, A.M. Pereira, R.L. Hadimani, D.C. Jiles, R.L. Hadimani, D.L. Schlagel, T.A. Lograsso, D.C. Jiles, and T.A. Lograsso: Suppression of magnetostructural transition on GdSiGe thin film after thermal cyclings. Thin Solid Films 621, 247-252 (2017).

68. A.L. Pires, J.H. Belo, I.T. Gomes, R.L. Hadimani, D.C. Jiles, L. Fernandes, P.B. Tavares, J.P. Araújo, A.M.L. Lopes, and A.M. Pereira: Annealing influence on the magnetostructural transition in $\mathrm{Gd}_{5} \mathrm{Si}_{1.3} \mathrm{Ge}_{2.7}$ thin films. Mater. Lett. 159, 301304 (2015)

69. J.M. Lommel: Magnetic and electrical properties of FeRh thin films. J. Appl. Phys. 37, 1483 (1966).

70. T. Zhou, M.K. Cher, L. Shen, J.F. Hu, and Z.M. Yuan: On the origin of giant magnetocaloric effect and thermal hysteresis in multifunctional $\alpha$-FeRh thin films. Phys. Lett. A 377, 3052-3059 (2013).

71. R.O. Cherifi, V. Ivanovskaya, L.C. Phillips, A. Zobelli, I.C. Infante, E. Jacquet, V. Garcia, S. Fusil, P.R. Briddon, N. Guiblin, A. Mougin, A.A. Ünal, F. Kronast, S. Valencia, B. Dkhil, A. Barthélémy, and M. Bibes: Electric-field control of magnetic order above room temperature. Nat. Mater 13, 345-351 (2014).

72. X. Moya, L.E. Hueso, F. Maccherozzi, A.I. Tovstolytkin, D.I. Podyalovskii, C. Ducati, L.C. Phillips, M. Ghidini, O. Hovorka, A. Berger, M.E. Vickers, E. Defay, S.S. Dhesi, and N.D. Mathur: Giant and reversible extrinsic magnetocaloric effects in $\mathrm{La}_{0.7} \mathrm{Ca}_{0.3} \mathrm{MnO}_{3}$ films due to strain. Nat. Mater. 12 52-58 (2013)

73. V. Uhlír, J.A. Arregi, and E.E. Fullerton: Colossal magnetic phase transition asymmetry in mesoscale FeRh stripes. Nat. Commun. 7, 13113 (2016).

74. A. Hillion, A. Cavallin, S. Vlaic, A. Tamion, F. Tournus, G. Khadra, J. Dreiser, C. Piamonteze, F. Nolting, S. Rusponi, K. Sato, T.J. Konno, O. Proux, V. Dupuis, and H. Brune: Low temperature ferromagnetism in chemically ordered $\mathrm{FeRh}$ nanocrystals. Phys. Rev. Lett. 110, 1-5 (2013).

75. C. Kuhrt, T. Schittny, and K. Barner: Magnetic B-T phase diagram of anion substituted MnAs magnetocaloric experiments. Phys. Status solidi 91, 105-113 (1985).

76. B.T.M. Willis and H.P. Rooksby: Magnetic transitions and structural changes in hexagonal manganese compounds. Proc. Phys. Soc. Sect. B 67, 290-296 (1954).

77. S. Gama, A.A. Coelho, A. De Campos, A. Magnus, G. Carvalho, F.C.G. Gandra, P.J. Von Ranke, and N.A. De Oliveira: Pressureinduced colossal magnetocaloric effect in MnAs. Phys. Rev. Lett. 93, 237202 (2004)

78. J.S. Amaral and V.S. Amaral: The effect of magnetic irreversibility on estimating the magnetocaloric effect from magnetization measurements. Appl. Phys. Lett. 94, 42506 (2009).

79. L. Caron, Z.Q. Ou, T.T. Nguyen, D.T. Cam Thanh, O. Tegus, and E. Bruck: On the determination of the magnetic entropy 
change in materials with first-order transitions. J. Magn. Magn. Mater. 321, 3559-3566 (2009).

80. D. Mosca, F. Vidal, and V. Etgens: Strain engineering of the magnetocaloric effect in MnAs epilayers. Phys. Rev. Lett. 101, 125503 (2008)

81. V. Kaganer, B. Jenichen, F. Schippan, W. Braun, L. Däweritz, and K. Ploog: Strain-mediated phase coexistence in MnAs heteroepitaxial films on GaAs: An X-ray diffraction study. Phys. Rev. B 66, 045305 (2002).

82. M. Trassinelli, M. Marangolo, M. Eddrief, V.H. Etgens, V. Gafton, S. Hidki, E. Lacaze, E. Lamour, C. Prigent, J-P. Rozet, S. Steydli, Y. Zheng, and D. Vernhet: Suppression of the thermal hysteresis in magnetocaloric MnAs thin film by highly charged ion bombardment. Appl. Phys. Lett. 104, 081906 (2014).

83. J.Y. Duquesne, J.Y. Prieur, J. Agudo Canalejo, V. Etgens, M. Eddrief, A. Ferreiram, and M. Marangolo: Ultrasonic triggering of giant magnetocaloric effect in MnAs thin films. Phys. Rev. B 86, 035207 (2012).

84. C. Spezzani, E. Ferrari, E. Allaria, F. Vidal, A. Ciavardini, R. Delaunay, F. Capotondi, E. Pedersoli, M. Coreno, C. Svetina, L. Raimondi, M. Zangrando, R. Ivanov, I. Nikolov, A. Demidovich, M.B. Danailov, H. Popescu, M. Eddrief, G. De Ninno, M. Kiskinov, and M. Sacchi: Magnetization and microstructure dynamics in $\mathrm{Fe} / \mathrm{MnAs} / \mathrm{GaAs}(001)$ : Fe magnetization reversal by a femtosecond laser pulse. Phys. Rev. Lett. 2, 113 (2014).

85. J. Lyubina, R. Schaefer, N. Martin, L. Schultz, and O. Gutfleisch: Novel design of $\mathrm{La}(\mathrm{Fe}, \mathrm{Si})_{13}$ alloys towards high magnetic refrigeration performance. Adv. Mater. 22, 3735+ (2010).

86. J. Lyubina: Recent advances in the microstructure design of materials for near room temperature magnetic cooling (invited). J. Appl. Phys. 109, 07A902-1-07A902-6 (2011).

87. M. Phejar, V. Paul-Boncour, and L. Bessais: Structural and magnetic properties of magnetocaloric $\mathrm{LaFe}_{13-x} \mathrm{Si}_{x}$ compounds synthesized by high energy ball-milling. Intermetallics $\mathbf{1 8}$, 2301-2307 (2010).

88. J.J. Ipus, J.M. Borrego, L.M. Moreno-Ramírez, J.S. Blázquez, V. Franco, and A. Conde: Grinding and particle size selection as a procedure to enhance the magnetocaloric response of $\mathrm{La}$ $(\mathrm{Fe}, \mathrm{Si})_{13}$ bulk samples. Intermetallics $\mathbf{8 4}, 30-34$ (2017).

89. F.X. Hu, L. Chen, J. Wang, L.F. Bao, J.R. Sun, and B.G. Shen: Particle size dependent hysteresis loss in $\mathrm{La}_{0.7} \mathrm{Ce}_{0.3} \mathrm{Fe}_{11.6} \mathrm{Si}_{1.4} \mathrm{C}_{0.2}$ first-order systems. Appl. Phys. Lett. 100, 072403 (2012).

90. W. Ligang and O. Tegus: Phase Formation and magnetocaloric effect in $\mathrm{La}\left(\mathrm{Fe}_{1-x}-\mathrm{Co}_{x}\right)_{11.2} \mathrm{Si}_{1.8}$ compounds prepared by ballmilling. Rare Met. Mater. Eng. 45, 2814-2817 (2016).

91. J. Huang, N. Sun, C. Liu, Y. Ge, T. Zhang, F. Liu, and P. Si: Effect of particle size on the hysteretic behavior and magnetocaloric effect of $\mathrm{La}_{0.5} \mathrm{Pr}_{0.5} \mathrm{Fe}_{11.4} \mathrm{Si}_{1.6}$ compound. Acta Metall. Sin. 27, 27-30 (2014).

92. X. Zhang, Y. Long, E. Niu, X.P. Shao, J. Shen, H. Zhang, Y. Long, E. Niu, X.P. Shao, J. Shen, F.X. Hu, J.R. Sun, and B.G. Shen: Influence of particle size on the hydrogenation in $\mathrm{La}(\mathrm{Fe}, \mathrm{Si})_{13}$ compounds. J. Appl. Phys. 911, 1-4 (2013).

93. O. Gutfleisch, T. Gottschall, M. Fries, D. Benke, I. Radulov, K.P. Skokov, H. Wende, M. Gruner, M. Acet, P. Entel, and M. Farle: Mastering hysteresis in magnetocaloric materials. Philos. Trans. R. Soc., A 374, 20150308 (2016).

94. J. Lanzarini, T. Barriere, M. Sahli, J.C. Gelin, A. Dubrez, C. Mayer, M. Pierronnet, and P. Vikner: Thermoplastic filled with magnetocaloric powder. Mater. Des. 87, 1022-1029 (2015).

95. H. Zhang, Y. Sun, Y. Li, Y. Wu, Y. Long, J. Shen, F. Hu, J. Sun, and B. Shen: Mechanical properties and magnetocaloric effects in $\mathrm{La}(\mathrm{Fe}, \mathrm{Si})_{13}$ hydrides bonded with different epoxy resins. J. Appl. Phys. 117, 1-5 (2015).
96. J. Feng, R. Geutjens, N.V. Thang, J. Li, X. Guo, A. Kéri, S. Basak, G. Galbács, G. Biskos, H. Nirschl, H.W. Zandbergen, E. Brück, and A. Schmidt-Ott: Magnetic phase transition in spark-produced ternary LaFeSi nanoalloys. ACS Appl. Mater. Interfaces 10, 6073-6078 (2018).

97. O. Tegus, E. Bruck, K.H.J. Buschow, and F.R. de Boer: Transition-metal-based magnetic refrigerants for roomtemperature applications. Nature 415, 150-152 (2002).

98. N.H. Dung, L. Zhang, Z.Q. Ou, and E. Brck: From first-order magneto-elastic to magneto-structural transition in $(\mathrm{Mn}, \mathrm{Fe})$ ${ }_{1.95} \mathrm{P}_{0.50} \mathrm{Si}_{0.50}$ compounds. Appl. Phys. Lett. 99, $2-4$ (2011).

99. N.H. Dung, Z.Q. Ou, L. Caron, L. Zhang, D.T.C. Thanh, G.A. de Wijs, R.A. de Groot, K.H.J. Buschow, and E. Brück: Mixed magnetism for refrigeration and energy conversion. Adv. Energy Mater. 1, 1215-1219 (2011).

100. C.M. Lukehart, S.B. Milne, S.R. Stock, R.D. Shull, and J.E. Wittig: Nanocomposites containing nanoclusters of $\mathrm{Fe}_{z} \mathrm{P}$ or $\gamma-\mathrm{Fe}_{2} \mathrm{O}_{3}$. Mater. Sci. Eng., A 5093, 176-180 (1995).

101. J. Park, B. Koo, Y. Hwang, C. Bae, K. An, J. Park, and H.M. Park: Novel synthesis of magnetic $\mathrm{Fe}_{2} \mathrm{P}$ nanorods from thermal decomposition of continuously delivered precursors using a syringe pump. Angew. Chem., Int. Ed. Engl. 116, 2332-2335 (2004).

102. Z. Pan, R. Wang, J. Li, S. Iqbal, and W. Liu: $\mathrm{Fe}_{2} \mathrm{P}$ nanoparticles as highly efficient freestanding co-catalyst for photocatalytic hydrogen evolution. Int. J. Hydrogen Energy 1-9, 5337-5345 (2017).

103. Y. Zhang, H. Zhang, Y. Feng, L. Liu, and Y. Wang: Unique $\mathrm{Fe}_{2} \mathrm{P}$ nanoparticles enveloped in sandwichlike graphited carbon sheets as excellent hydrogen evolution reaction catalyst and lithium-ion battery anode. ACS Appl. Mater. Interfaces 7, 26684-26690 (2015).

104. D.E. Schipper, Z. Zhao, H. Thirumalai, A.P. Leitner, S.L. Donaldson, A. Kumar, F. Qin, Z. Wang, L.C. Grabow, J. Bao, and K.H. Whitmire: Effects of catalyst phase on the hydrogen evolution reaction of water splitting: Preparation of phase-pure films of effects of catalyst phase on the hydrogen evolution reaction of water splitting. Chem. Mater. 30, 3588-3598 (2018).

105. A.A.P. Leitner, D.E. Schipper, J. Chen, A.C. Colson, I. Rusakova, B.K. Rai, and K.H. Whitmire: Synthesis of hexagonal FeMnP thin films from a single-source molecular precursor. Chem. - A Eur. J. 23, 5565-5572 (2014).

106. M. Shirani and M. Kadkhodaei: Constitutive modeling of NiMn-Ga ferromagnetic shape memory alloys under biaxial compression. J. Intell. Mater. Syst. Struct. 27, 1547-1564 (2016).

107. K. Ullakko, J.K. Huang, C. Kantner, R.C. O'Handley, and V.V. Kokorin: Large magnetic-field-induced strains in $\mathrm{Ni}_{2} \mathrm{MnGa}$ single crystals. Appl. Phys. Lett. 69, 1966 (1996).

108. P. Ranzieri, S. Fabbrici, L. Nasi, L. Righi, F. Casoli, V.A. Chernenko, E. Villa, and F. Albertini: Epitaxial Ni-Mn$\mathrm{Ga} / \mathrm{MgO}(100)$ thin films ranging in thickness from 10 to $100 \mathrm{~nm}$. Acta Mater. 61, 263-272 (2013).

109. A. Planes, L. Mañosa, and M. Acet: Magnetocaloric effect and its relation to shape-memory properties in ferromagnetic Heusler alloys. J. Phys. Condens. Matter 21, 233201 (2009).

110. C. Wang, J. Meyer, N. Teichert, A. Auge, E. Rausch, B. Balke, A. Hütten, G.H. Fecher, and C. Felser: Heusler nanoparticles for spintronics and ferromagnetic shape memory alloys. J. Vac. Sci. Technol. B 32, 020802 (2014).

111. N. Teichert, A. Auge, E. Yüzüak, I. Dincer, Y. Elerman, B. Krumme, H. Wende, O. Yildirim, K. Potzger, and A. Hütten: Influence of film thickness and composition on the martensitic transformation in epitaxial $\mathrm{Ni}-\mathrm{Mn}-\mathrm{Sn}$ thin films Acta Mater. 86, 279-285 (2015). 
112. D.C. Dunand and P. Müllner: Size effects on magnetic actuation in Ni-Mn-Ga shape-memory alloys. Adv. Mater. 23, 216-232 (2011).

113. M. Chmielus, X.X. Zhang, C. Witherspoon, D.C. Dunand, and P. Müllner: Giant magnetic-field-induced strains in polycrystalline Ni-Mn-Ga foams. Nat. Mater. 8, 863-866 (2009).

114. M. Thomas and O. Heczko: Magnetic field-induced twin boundary motion in polycrystalline Ni-Mn-Ga fibres. New J. Phys. 10, 073002 (2008).

115. M. Potschke, S. Weiss, U. Gaitzsch, D. Cong, C. Hu, S. Roth, and L. Schultz: Magnetically resettable $0.16 \%$ free strain in polycrystalline Ni-Mn-Ga plates. Scr. Mater. 63, 383-386 (2010).

116. A.L. Roytburd, T.S. Kim, Q. Su, J. Slutsker, and M. Wuttig: Martensitic transformation in constrained films. Acta Mater. 46, 5095-5107 (1998).

117. G.A. Malygin: Size effects under martensitic deformation of shape-memory alloys. Tech. Phys. 54, 1782-1785 (2009).

118. S. Vinodh Kumar, R.K. Singh, S. Seenithurai, S. Bysakh, M. Manivel Raja, and M. Mahendran: Phase structure and magnetic properties of the annealed Mn-rich Ni-Mn-Ga ferromagnetic shape memory thin films. Mater. Res. Bull. 61, 95-100 (2015).

119. J. Buschbeck, R. Niemann, O. Heczko, M. Thomas, L. Schultz, and S. Fähler: Situ studies of the martensitic transformation in epitaxial Ni-Mn-Ga films. Acta Mater. 57, 2516-2526 (2009).

120. M. Thomas, O. Heczko, J. Buschbeck, U.K. Rößler, J. McCord, N. Scheerbaum, L. Schultz, and S. Fähler: Magnetically induced reorientation of martensite variants in constrained epitaxial Ni$\mathrm{Mn}-\mathrm{Ga}$ films grown on $\mathrm{MgO}(001)$. New J. Phys. 10, 023040 (2008).

121. H.S. Akkera, N. Choudhary, and D. Kaur: Martensitic phase transformations and magnetocaloric effect in $\mathrm{Al}$ co-sputtered $\mathrm{Ni}-$ Mn-Sb alloy thin films. Mater. Sci. Eng. B 198, 113-119 (2015).

122. Y. Liu and X. Huang: Substrate-induced stress and transformation characteristics of a deposited $\mathrm{Ti}-\mathrm{Ni}-\mathrm{Cu}$ thin film. Philos. Mag. 84, 1919-1936 (2004).

123. R. Vishnoi, R. Singhal, and D. Kaur: Thickness dependent phase transformation of magnetron-sputtered $\mathrm{Ni}-\mathrm{Mn}-\mathrm{Sn}$ ferromagnetic shape memory alloy thin films. J. Nanoparticle Res. 13, 39753990 (2011).

124. M. Kallmayer, P. Pörsch, T. Eichhorn, H. Schneider, C.A. Jenkins, G. Jakob, and H.J. Elmers: Compositional dependence of element-specific magnetic moments in $\mathrm{Ni}_{2} \mathrm{MnGa}$ films. J. Phys. D: Appl. Phys. 42, 084008 (2009).

125. O. Heczko, M. Thomas, J. Buschbeck, L. Schultz, and S. Fähler: Epitaxial Ni-Mn-Ga films deposited on $\mathrm{SrTiO}_{3}$ and evidence of magnetically induced reorientation of martensitic variants at room temperature. Appl. Phys. Lett. 92, 072502-1-072502-3 (2008).

126. Y. Zhang, R.A. Hughes, J.F. Britten, J.S. Preston, G.A. Botton, and M. Niewczas: Self-activated reversibility in the magnetically induced reorientation of martensitic variants in ferromagnetic Ni-Mn-Ga films. Phys. Rev. B: Condens. Matter Mater. Phys. 81, 054406-9-1-054406-9 (2010).

127. T.J. Zhu, L. Lu, M.O. Lai, and J. Ding: Growth and magnetic properties of $\mathrm{NiMnGa}$ thin films prepared by pulsed laser ablation. Smart Mater. Struct. 14, S293-S296 (2005).

128. M. Schubert, H. Schaefer, J. Mayer, A. Laptev, M. Hettich, M. Merklein, C. He, C. Rummel, O. Ristow, M. Großmann, Y. Luo, V. Gusev, K. Samwer, M. Fonin, T. Dekorsy, and J. Demsar: Collective modes and structural modulation in $\mathrm{Ni}-$ $\mathrm{Mn}-\mathrm{Ga}(\mathrm{Co})$ martensite thin films probed by femtosecond spectroscopy and scanning tunneling microscopy. Phys. Rev. Lett. 115, 076402 (2015).
129. Y. Zhang, R.A. Hughes, J.F. Britten, P.A. Dube, J.S. Preston, G.A. Botton, and M. Niewczas: Magnetocaloric effect in $\mathrm{Ni}-$ Mn-Ga thin films under concurrent magnetostructural and Curie transitions. J. Appl. Phys. 110, 013910 (2011).

130. V. Recarte, J.I. Pérez-Landazábal, V. Sánchez-Alárcos, V.A. Chernenko, and M. Ohtsuka: Magnetocaloric effect linked to the martensitic transformation in sputter-deposited Ni-Mn-Ga thin films. Appl. Phys. Lett. 95, 141908 (2009).

131. N. Teichert, D. Kucza, O. Yildirim, E. Yuzuak, I. Dincer, A. Behler, B. Weise, L. Helmich, A. Boehnke, S. Klimova, A. Waske, Y. Elerman, and A. Hütten: Structure and giant inverse magnetocaloric effect of epitaxial $\mathrm{Ni}-\mathrm{Co}-\mathrm{Mn}-\mathrm{Al}$ films. Phys. Rev. B 91, 184405-1-184405-7 (2015).

132. R. Niemann, O. Heczko, L. Schultz, and S. Fähler: Metamagnetic transitions and magnetocaloric effect in epitaxial Ni-Co-Mn-In films. Appl. Phys. Lett. 97, 222507-1-222507-3 (2010).

133. K. Zhang, J. Sunarso, Z. Shao, W. Zhou, C. Sun, and S. Liu: Research progress and materials selection guidelines on mixed conducting perovskite-type ceramic membranes for oxygen production. RSC Adv. 1, 1661-1676 (2011).

134. V.M. Goldschmidt: Die Gesetze der Krystallochemie, Vol. 14 (Springer-Verlag, Berlin, Germany, 1926).

135. A.M. Glazer: The classification of tilted octahedra in perovskites. Acta Crystallogr. Sect. B Struct. Crystallogr. Cryst. Chem. 28 3384-3392 (1972).

136. J.B. Goodenough: Theory of the role of covalence in the perovskite-type manganites $[\mathrm{La}, \mathrm{M}(\mathrm{II})] \mathrm{MnO}_{3}$. Phys. Rev. 100, 564-573 (1955).

137. J. Kanamori: Superexchange interaction and symmetry properties of electron orbitals. J. Phys. Chem. Solids 10, 87-98 (1959).

138. P.W. Anderson: Antiferromagnetism. Theory of superexchange interaction. Phys. Rev. 79, 350-356 (1950).

139. S. Jin, T.H. Tiefel, M. McCormack, R.A. Fastnacht, R. Ramesh, and L.H. Chen: Thousandfold change in resistivity in magnetoresistive La-Ca-Mn-O films. Science 264, 413-415 (1994).

140. P. Lampen, N.S. Bingham, M.H. Phan, H. Kim, M. Osofsky, A. Piqué, T.L. Phan, S.C. Yu, and H. Srikanth: Impact of reduced dimensionality on the magnetic and magnetocaloric response of $\mathrm{La}_{0.7} \mathrm{Ca}_{0.3} \mathrm{MnO}_{3}$. Appl. Phys. Lett. 102, 062414 (2013).

141. Z.M. Wang, T. Tang, Y.P. Wang, S.Y. Zhang, and Y.W. Du: Room temperature large magnetoresistance and magnetocaloric properties of $\mathrm{La}_{0.78} \mathrm{Ag}_{0.22} \mathrm{MnO}_{3}$ film. J. Magn. Magn. Mater. 246, 254-258 (2002).

142. M. Koubaa, W.C.R. Koubaa, and A. Cheikhrouhou: Magnetocaloric effect and magnetic properties of $\mathrm{La}_{0.75} \mathrm{Ba}_{0.1} \mathrm{M}_{0.15} \mathrm{MnO}_{3}$ $(\mathrm{M}=\mathrm{Na}, \mathrm{Ag}$, and $\mathrm{K})$ perovskite manganites. J. Alloys Compd. 479, 65-70 (2009)

143. W. Prellier, P. Lecoeur, and B. Mercey: Colossal-magnetoresistive manganite thin films. J. Phys. Condens. Matter 13, R915 (2001)

144. D.D. Belyea, T.S. Santos, and C.W. Miller: Magnetocaloric effect in epitaxial $\mathrm{La}_{0.56} \mathrm{Sr}_{0.44} \mathrm{MnO}_{3}$ alloy and digital heterostructures. J. Appl. Phys. 111, 07A935 (2012).

145. A. Goktas, I.H. Mutlu, and A. Kawashi: Growth and characterization of $\mathrm{La}_{1-x} \mathrm{~A}_{x} \mathrm{MnO}_{3}(\mathrm{~A}=\mathrm{Ag}$ and $\mathrm{K}, x=0.33)$ epitaxial and polycrystalline manganite thin films derived by sol-gel dip-coating technique. Thin Solid Films 520, 6138-6144 (2012).

146. V.S. Kumar, R. Chukka, Z. Chen, P. Yang, and L. Chen: Strain dependent magnetocaloric effect in $\mathrm{La}_{0.67} \mathrm{Sr}_{0.33} \mathrm{MnO}_{3}$ thin-films AIP Adv. 3, 052127 (2013).

147. S.K. Giri, P. Dasgupta, A. Poddar, R.C. Sahoo, D. Paladhi, and T.K. Nath: Strain modulated large magnetocaloric effect in $\mathrm{Sm}_{0.55} \mathrm{Sr}_{0.45} \mathrm{MnO}_{3}$ epitaxial films epitaxial films. Appl. Phys. Lett. 106, 023507 (2015). 
148. B. Kundys and H. Szymczak: Magnetostriction in thin films of manganites and cobaltites. Phys. Status Solidi 201, 3247-3251 (2004).

149. R.E.E. Rosensweig: Heating magnetic fluid with alternating magnetic field. J. Magn. Magn. Mater. 252, 370-374 (2002).

150. R.E. Rosensweig: Ferrohydrodynamics (Cambridge University Press, 1985).

151. G.V. Brown: Magnetic heat pumping near room temperature. $J$. Appl. Phys. 47, 3673-3680 (1976).

152. J.E. Burnett: Magnetic Heating and Cooling Systems. Patent No. 5231 834, August 3, 1993.

153. P. Shirron, E. Canavan, M. DiPirro, M. Jackson, T. King, J. Panek, and J. Tuttle: A compact, high-performance continuous magnetic refrigerator for space missions. Cryogenics 41, 789795 (2001).

154. R.G. Ross, Jr.: Cryocoolers 13, Vol. 53 (Kluwer Academic/ Plenum Publishers, Dortecht, The Netherlands, 2013).

155. U. Tomc, J. Tušek, A. Kitanovski, and A. Poredoš: A new magnetocaloric refrigeration principle with solid-state thermoelectric thermal diodes. Appl. Therm. Eng. 58, 1-10 (2013).
156. J.A. Turcaud, K. Morrison, A. Berenov, N.M. Alford, K.G. Sandeman, and L.F. Cohen: Microstructural control and tuning of thermal conductivity in $\mathrm{La}_{0.67} \mathrm{Ca}_{0.33} \mathrm{MnO}_{3 \pm \delta}$. Scr. Mater. 68, 510-513 (2013).

157. A. Davarpanah, J.S. Amaral, and V.S. Amaral: Thermal conductivity of magnetocaloric materials: Temperature dependence and its impact on magnetocaloric cycles (in press).

158. V.H. Dave, H.A. Virpura, and S.P. Bhatnagar: Thermal liquid propulsion system using magnetic nanofluid. AIP Conf. Proc. 1961, 1-3 (2018).

159. V. Chaudhary, Z. Wang, A. Ray, I. Sridhar, and R.V. Ramanujan: Self pumping magnetic cooling. J. Phys. D. Appl. Phys. 50, 03LT03 (2017).

160. M. Cleveland and H. Liang: Magnetocaloric piezoelectric composites for energy harvesting. Smart Mater. Struct. 21, 47002 (2012).

161. B. Barkley, C. Sanchez, and H. Liang: Situ strengthening of the aluminum-based gadolinium alloy composite for tribological applications. J. Tribol. 134, 11603 (2012).

162. M.R. Barati, C. Selomulya, K.G. Sandeman, and K. Suzuki: Extraordinary induction heating effect near the first order Curie transition. Appl. Phys. Lett. 105, 4-9 (2014). 